\title{
Modélisation du plancton dans une retenue oligotrophe : Sainte-Croix sur le Verdon.
}

\author{
Anne-Marie Pourcher (1) et Marie-José Salençon (2). \\ (1) Institut Pasteur de Lille. \\ (2) EDF, Département EAA, 6, quai Watier. 78401 Chatou Cedex.
}

Résumé. - Un modèle biologique a été développé en vue de représenter le fonctionnement de l'écosystème planctonique dans la retenue oligotrophe de Sainte-Croix. II a une structure physique simplifiée (modèle bicouche) qui simule la stratification thermique saisonnière.

Ce modèle a représenté correctement les principales poussées planctoniques. L'étude a également mis en évidence l'importance de la fermeture du modèle : il s'agit de la prédation par le zooplancton dans ce cas. Le rôle primordial des variables physiques (température, stratification thermique verticale), notamment dans l'évolution du stock de nutriments, confirme la nécessité de coupler les processus physiques et biologiques dans un modèle écologique.

Summary. - A biological model has been developed to portray the planckton ecosystem dynamics in the oligotrophic reservoir of Sainte-Croix. Its simplified physical structure (two-layers model) simulates seasonal thermal stratification.

The model has portrayed the main planktonic growths with accuracy. The study has also revealed the importance of closing the model, in this case, for to take zooplanktonic predation into account. The leading role of physical variables (temperature, vertical thermal stratification), in particular in the evolution of the nutrient stock, confirms the need to couple physical and biological processes in an ecological model.

Ce travail a été réalisé avec la collaboration de l'équipe d'Hydrobiologie de l'Université de Provence (MM. Champeau, Pont et Vaquer) qui a assuré la collecte des données et a apporté l'appui scientifique en écologie, ainsi qu'avec les conseils de M. Thebault (Université Paul Sabatier) pour la modélisation biologique. 


\section{I - INTRODUCTION.}

Un écosystème lacustre est un ensemble complexe où interviennent des phénomènes physiques, physicochimiques et biologiques. Si l'approche par la mesure permet de mieux comprendre son fonctionnement, elle ne permet pas toujours de prévoir son évolution sous diverses contraintes.

Aussi a-t-on recours à des outils prévisionnels comme les modèles mathématiques.

Les modèles de simulation du plancton dans les réservoirs ont pour objet de décrire et de prévoir l'évolution de la qualité physico-chimique et biologique des eaux.

L'objectif de la présente étude consiste à mettre au point la partie physico-chimique et biologique de la modélisation de la retenue hydroélectrique de Sainte-Croix sur le Verdon en s'appuyant sur un modèle physique simple: un modèle bicouche.

Après une analyse des données de terrain, le modèle sera construit et ajusté sur l'échantillon de mesures disponibles.

Une étude de sensibilité permettra ensuite d'estimer l'importance relative des paramètres de la modélisation sur la réponse de l'écosystème simulé.

On trouvera dans le rapport détaillé de l'étude (Pourcher et Salençon, 1988), l'ensemble des données et des résultats dont une présentation synthétique est faite dans cet article.

\section{2 - ELABORATION DU MODELE BIOLOGIQUE DE SAINTE-CROIX.}

\subsection{Caractéristiques hydrauli- ques et thermiques.}

Située sur le cours du Verdon à $109 \mathrm{~km}$ de sa source, cette retenue est l'une des plus grandes de France $\left(703 \mathrm{Mm}^{3}\right.$ ) avec une superficie de $22 \mathrm{~km}^{2}$ et une profondeur moyenne de $50 \mathrm{~m}$ (maximum de $83 \mathrm{~m}$ au barrage) (fig. 1). Ce réservoir est caractérisé par:

- un temps de renouvellement long (280 jours),

- les apports de 2 rivières (le Verdon et le Maire) et d'une résurgence (Fontaine l'Evêque),

- un fonctionnement hydraulique en station de pompage où alternent au barrage les phases de pompage et turbinage. La gestion annuelle fait apparaître un marnage hivernal important pouvant atteindre $16 \mathrm{~m}$.

Le lac se trouve dans une zone climatique de type méditerranéen subhumide et se définit comme un lac monomictique chaud: les eaux superficielles ont une température supérieure à $4^{\circ} \mathrm{C}$ (quelle que soit la saison) et leur réchauffement entraine la formation d'une thermocline stable en été.

\subsection{Structure du modèle.}

L'évolution de la structure thermique verticale d'un réservoir résulte des échanges atmosphériques et des ap- 


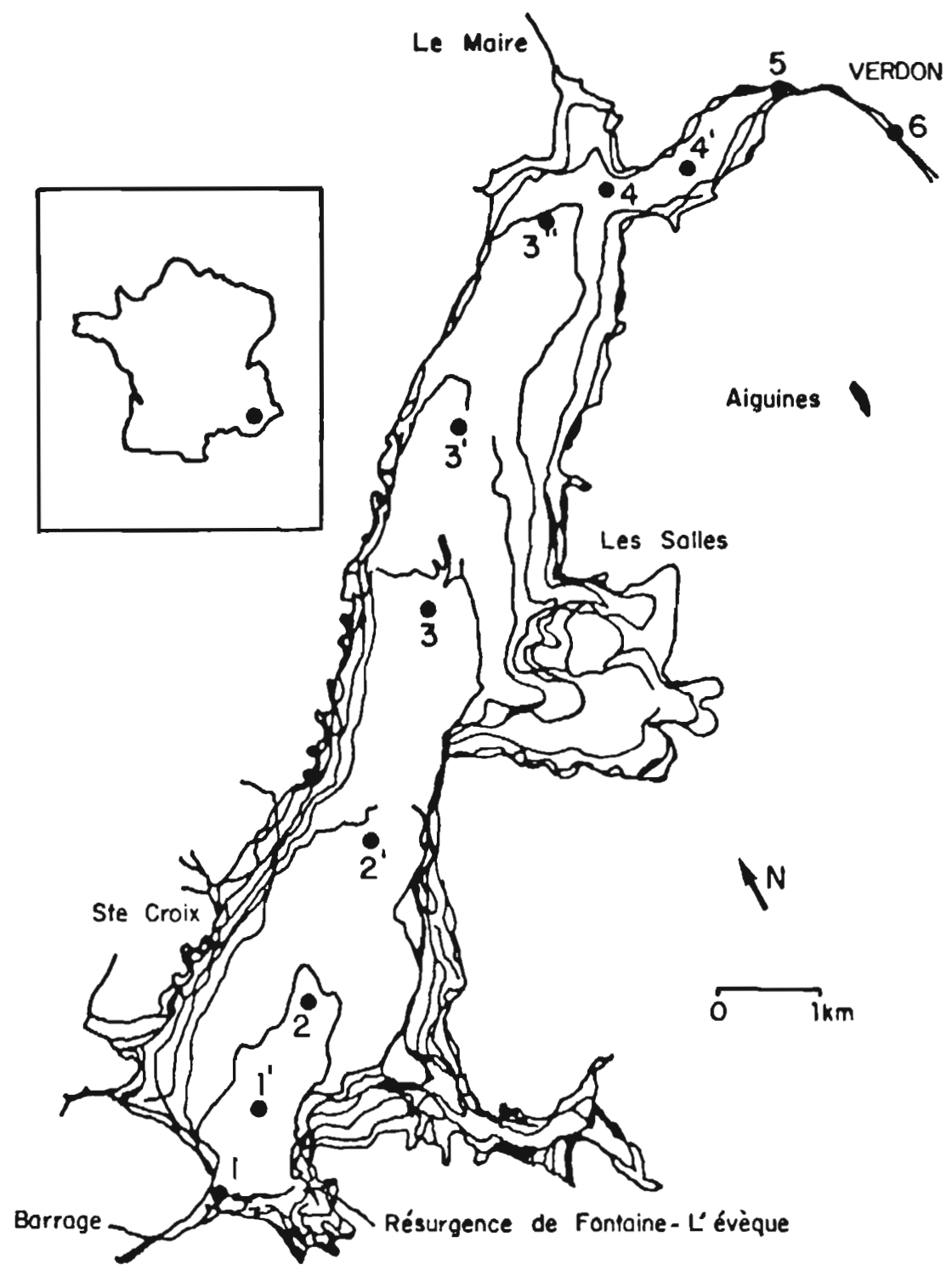

Fig. 1. - Le réservoir de Sainte-Croix; bathymétrie (courbes de niveau NGF équidistantes de $10 \mathrm{~m}$ ) et situation des stations de prélèvements. 
ports et retraits d'eau au réservoir. Cette structure verticale joue un rôle primordial sur l'écosystème car elle régit les mouvements internes des masses d'eau.

Dans nos régions, un lac est isotherme en hiver, à une valeur voisine de $4{ }^{\circ} \mathrm{C}$. Au printemps les couches de surface se réchauffent sous l'effet du rayonnement solaire. Le vent fournit l'énergie nécessaire au mélange de cette eau chaude de surface jusqu'à une certaine profondeur: c'est une thermocline transitoire. L'accumulation de ces petites thermoclines donne naissance à une thermocline saisonnière, stable en profondeur. Le réservoir est alors séparé en 3 zones dont une schématisation est proposée par Enderlé (1982) (fig. 2) :
L'épilimnion: couche de surface turbulente et bien mélangée en contact permanent avec l'extérieur par l'interface air-eau.

La thermocline: zone de fort gradient thermique qui se comporte comme une barrière de densité.

L'hypolimnion: couche inférieure qui est le siège de phénomènes diffusifs.

A l'automne, le refroidissement des couches superficielles engendre des mouvements convectifs qui provoquent un enfoncement progressif de la thermocline jusqu'à obtenir le mélange hivernal.

Ces phénomènes, très marqués dans un lac, sont perturbés par les phénomènes advectifs dans un réservoir: entrées d'eau naturelles (ri-

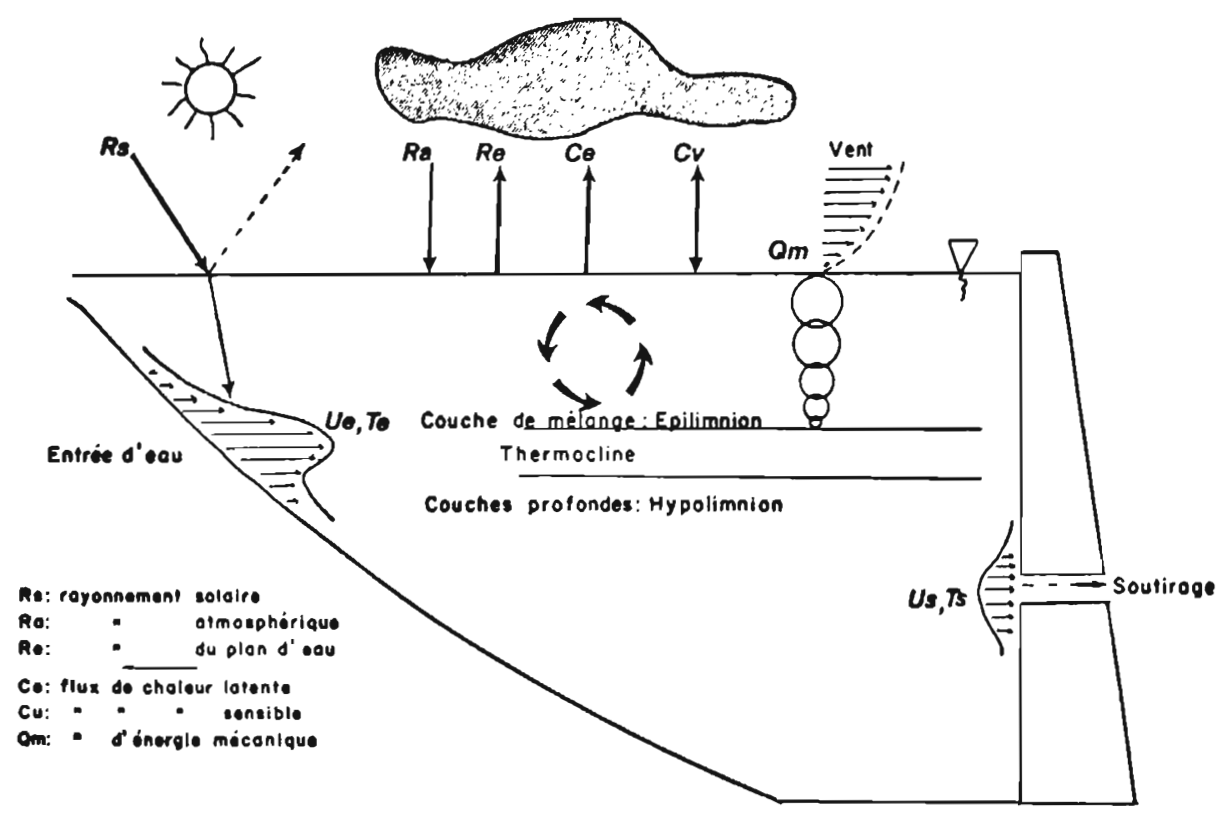

Fig. 2 - Bilan d'énergie d'une retenue et dynamique interne. 
vières, ruissellement...), entrées d'eau artificielles (injections en profondeur par des ouvrages d'amenée), soutirages.

La structure du sous-système physique retenue dans cette étude est très simple : c'est un modèle vertical bicouche. La masse d'eau est divisée en 2 couches considérées homogènes spatialement, qui représentent la stratification thermique saisonnière.

Une couche de sédiment est ajoutée pour stocker les algues et la matière organique qui sédimentent (fig. 3). La sédimentation est prise en compte dans le modèle par une vitesse constante et spécifique de chaque variable.

La couche de surface, l'épilimnion, est turbulente. Elle est séparée de la couche de fond non turbulente, l'hypolimnion, par une thermocline. Chaque couche est caractérisée par sa température, son volume, sa profondeur et l'intensité du rayonnement solaire à sa limite supérieure. La dynamique des 2 masses d'eau est "forcée" à partir des mesures de températures réalisées sur le site.

La stratification thermique saisonnière est représentée dans le modèle par l'évolution du volume et de la hauteur de chaque couche. La thermocline étant bien établie en été, les couches de surface et de fond sont indépendantes. En hiver, lorsque la stratification disparaît, la couche turbulente de surface atteint le fond. Une partie de la couche de sédiment est remise en suspension dans la masse d'eau.

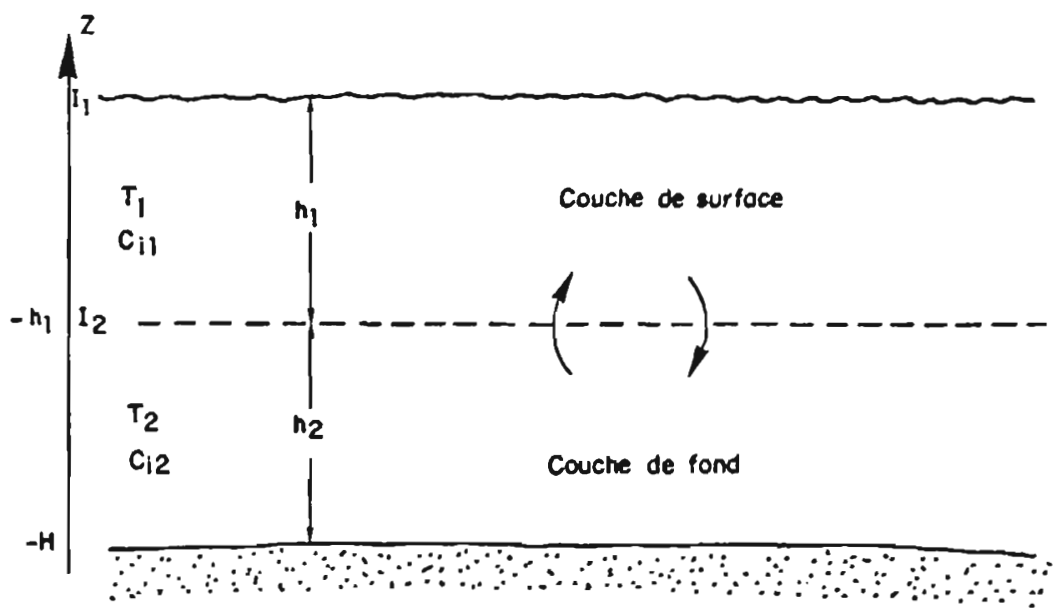

$l_{1}, l_{2}$ : intensité du rayonnement solaire au niveau de la limite supérieure de chaque couche. $T_{1}, T_{2}$ : température de la couche de surface (de fond).

$\mathrm{C}_{\mathrm{y}} \mathrm{C}_{\mathrm{i} 2}$ : concentration en élément $\mathrm{i}$ dans la couche de surface (de fond).

$H$ : profondeur de la colonne d'eau.

$h_{1}, h_{2}$ : profondeur de la couche de surface (de fond).

Fig. 3. - Structure verticale schématisée du modèle. 
Les entrées et sorties ne sont pas prises en compte, le temps de séjour étant suffisamment long (280 jours), pour que, dans ce premier modèle global de la physique du lac, celui-ci soit considéré fermé.

\section{Analyse des données de terrain.}

Les mesures faites sur le terrain permettent de préciser la composition du plancton, d'évaluer la concentration en sels nutritifs et de déterminer les valeurs des variables caractérisant chaque couche du lac.

La température de l'eau et l'intensité du rayonnement solaire sont mesurées régulièrement depuis 1982, la première par un système automatique de mesures situé en amont du barrage, la seconde par une station météorologique située à quelques $\mathrm{km}$ du lac.

En 1985 et 1986, 23 sorties ont été faites sur le site de Sainte-Croix afin de mesurer l'extinction de la lumière et de déterminer les caractéristiques du plancton. Bien que seules les données acquises en 1986 soient dépouillées, c'est cette poériode d'étude 1985-1986 que nous choisissons pour la simulation.

\section{La température.}

Son rôle est primordial, non seulement par son influence directe sur la croissance du plancton mais aussi parce que c'est elle qui régit les mouvements d'eau dans la retenue.

La température est mesurée toutes les 3 heures depuis la surface jus- qu'au fond du lac à différentes profondeurs (à $1 \mathrm{~m}$ sous la surface de l'eau, tous les 3 mètres de $4 \mathrm{~m}$ à $31 \mathrm{~m}$ puis tous les 5 mètres de $32 \mathrm{~m}$ à $82 \mathrm{~m}$ ). Les caractéristiques des deux couches sont déterminées à partir de ces profils de température moyennés sur une journée.

Les gradients de température sur la verticale nous permettent de déduire la hauteur de la thermocline et par conséquent la température moyenne des 2 couches ainsi que leur volume. Les gradients de température mesurés entre 2 profondeurs successives varient de 0,1 à $1^{\circ} \mathrm{C} / \mathrm{m}$ au cours de l'année. Les pompages du barrage (situé à proximité du système automatique de mesures) ont une influence sur la dynamique de la masse d'eau et peuvent expliquer les faibles valeurs du gradient. Si le gradient est inférieur à $0,3{ }^{\circ} \mathrm{C} / \mathrm{m}$, la masse d'eau est considérée mélangée, sinon la profondeur pour laquelle le gradient est maximum correspond à la hauteur de la thermocline.

Le lac est divisé sur la verticale en 22 tranches d'eau centrées autour des points de mesure. Chaque tranche est caractérisée par sa température (qui correspond à celle du point de mesure) et par son volume, évalué d'après la courbe de capacité de la retenue.

Les volumes de l'hypolimnion et de l'épilimnion sont déterminés en sommant les volumes des tranches d'eau (du fond à la termocline pour la couche inférieure et de la thermocline à la surface pour la couche supérieure). Leur température est calculée 
en moyennant les températures des 22 tranches d'eau, pondérées par leur volume.

Les années 1985 et 1986 présentent peu de valeurs manquantes ( 2 jours en 1985 et 8 jours en 1986). Ces données manquantes sont calculées par interpolation linéaire.

Les tracés des profondeurs de thermocline, des gradients et des températures moyennes des 2 couches pour les années 1981 à 1986 ont montré une stabilité annuelle des températures de l'hypolimnion : $9.8 \pm 1.9^{\circ} \mathrm{C}$ en 85 et $9.3 \pm 1.8^{\circ} \mathrm{C}$ en 86 , alors que l'épilimnion, directement soumis aux conditions météorologiques présente des variations saisonnières beaucoup plus importantes (de $9^{\circ} \mathrm{C}$ à $23^{\circ} \mathrm{C}$ ).

De 1981 à 1986, quelle que soit l'année, l'évolution de la thermocline est caractérisée par 4 grandes phases (fig. 4):

- un mélange de la masse d'eau de décembre à mars : mélange hivernal (I),
- une mise en place de la thermocline de mars à avril : période de transition (II),

- une stabilité de la thermocline de mai à juillet (III) à environ $12 \mathrm{~m}$ de profondeur, puis un enfoncement de celle-ci en août (III) à $16 \mathrm{~m}$ de profondeur,

- un enfoncement progressif de la thermocline de septembre à décembre (IV).

\section{Rayonnement solaire et coefficient d'extinction.}

- Les mesures du rayonnement solaire, trihoraires, sont moyennées sur la journée.

- Les sorties effectuées sur le site ont permis de mesurer la profondeur de disparition du disque de Secchi, indicateur de la transparence de l'eau, ainsi que la transmission de la lumière dans l'eau au moyen d'un quantamètre. La méthode du disque de Secchi et la mesure de la transmission lumineuse déterminent un coefficient d'extinction de la lu-

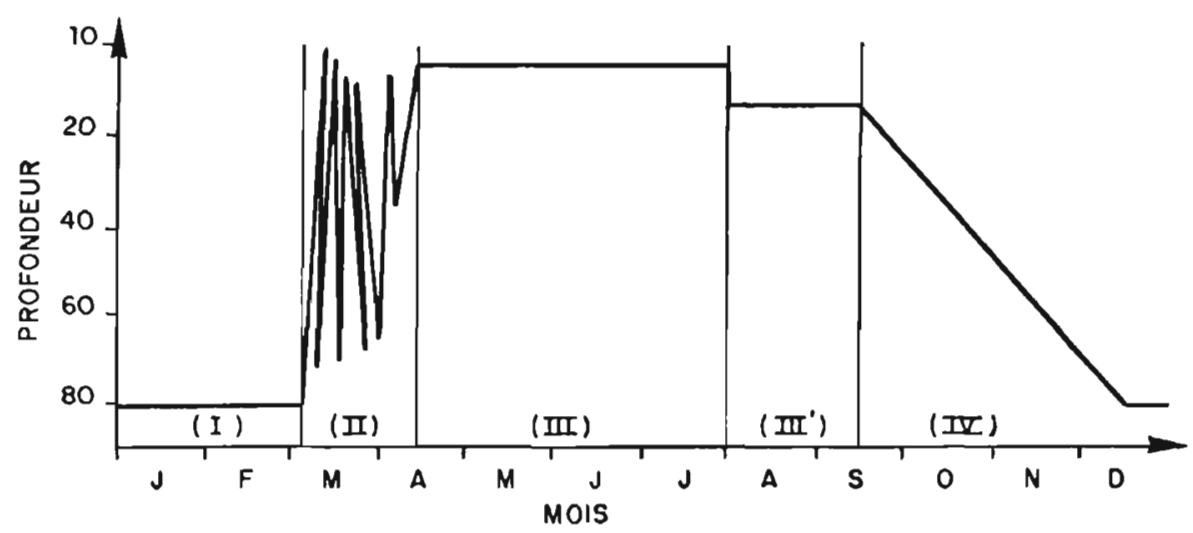

Fig. 4. - Schématisation de l'évolution de la thermocline. 
mière $(K)$ qui permet de calculer l'intensité lumineuse disponible à une profondeur donnée, notamment au niveau de la thermocline. Ces mesures dont d'autant plus importantes qu'elles permettent de déterminer la zone où la photosynthèse a lieu : couche euphotique dont la profondeur est délimitée par une intensité lumineuse égale à $1 \%$ de l'intensité incidente.

\section{"Disque de Secchi.}

La relation admise entre la mesure du disque de Secchi et le coefficient d'extinction est de la forme :

$\mathrm{K}=\frac{1,7}{\mathrm{~S}}$ où $\mathrm{S}$ représente la hauteur de disparition du disque (en mètres).

La profondeur de disparition du disque de Secchi $(S)$ varie régulièrement au cours des deux années. On observe une diminution progressive de la transparence durant la période de mélange : en 1985, de 7,5 $\mathrm{m}$ en janvier à $3,8 \mathrm{~m}$ en juin; en 1986, de $5,0 \mathrm{~m}$ en décembre à $2,2 \mathrm{~m}$ en mai. La transparence réaugmente rapidement dès le début de la période stratifiée. En 1985, le maximum est obtenu en septembre $(6,7 \mathrm{~m})$; en 1986, il apparaît plus tardivement au moment du brassage en décembre $(11,4 \mathrm{~m})$. Cette dernière valeur est exceptionnelle; c'est la plus élevée depuis 1982. Cette évolution du degré de la transparence des eaux s'éloigne du schéma classique puisque la diminution la plus grande de la transparence est notée en hiver : elle pourrait être liée aux importants apports de matières en provenance du Verdon à cette période.

\section{* Mesures au quantamètre.}

Les quantités de lumière transmise sont appréciées tous les mètres et sont traduites en pourcentage de lumière incidente mesurée à la surface. Globalement, les profils suivent une courbe classique d'extinction de la lumière comme le démontrent par ailleurs les très bons ajustements à la loi de Beer Lambert : $I Z=10 . e^{K Z}$

$\mathrm{IZ}$ : intensité lumineuse à la profondeur $\mathbf{Z}$

lo: intensité lumineuse en surface

$\mathrm{K}$ : coefficient d'extinction.

Les coefficients de corrélation ( $r$ ) sont toujours supérieurs à 0,95 (significatifs à plus de $1 \%$ ). Ce résultat traduit le caractère homogène de la couche d'eau du point de vue de ses qualités optiques.

La couche euphotique est généralement comprise dans la couche de surface.

Les calculs des coefficients d'extinction par la méthode du disque de Secchi et la mesure de la transmission lumineuse sont traités dans l'annexe 3 du rapport de Pourcher et Salençon (1988).

Les résultats (fig. 5) montrent que les valeurs des coefficients d'extinction déterminées par la $1^{\text {ère }}$ méthode diffèrent de celles obtenues à partir des mesures au quantamètre.

Par définition, la profondeur de disparition du disque de Secchi (S) correspond à un pourcentage de lumière transmise (IS) de l'ordre de $18 \%$ (cf. annexe 3 ), alors que les valeurs de IS mesurées à la hauteur de disparition du disque fluctuent de 1,7 à 


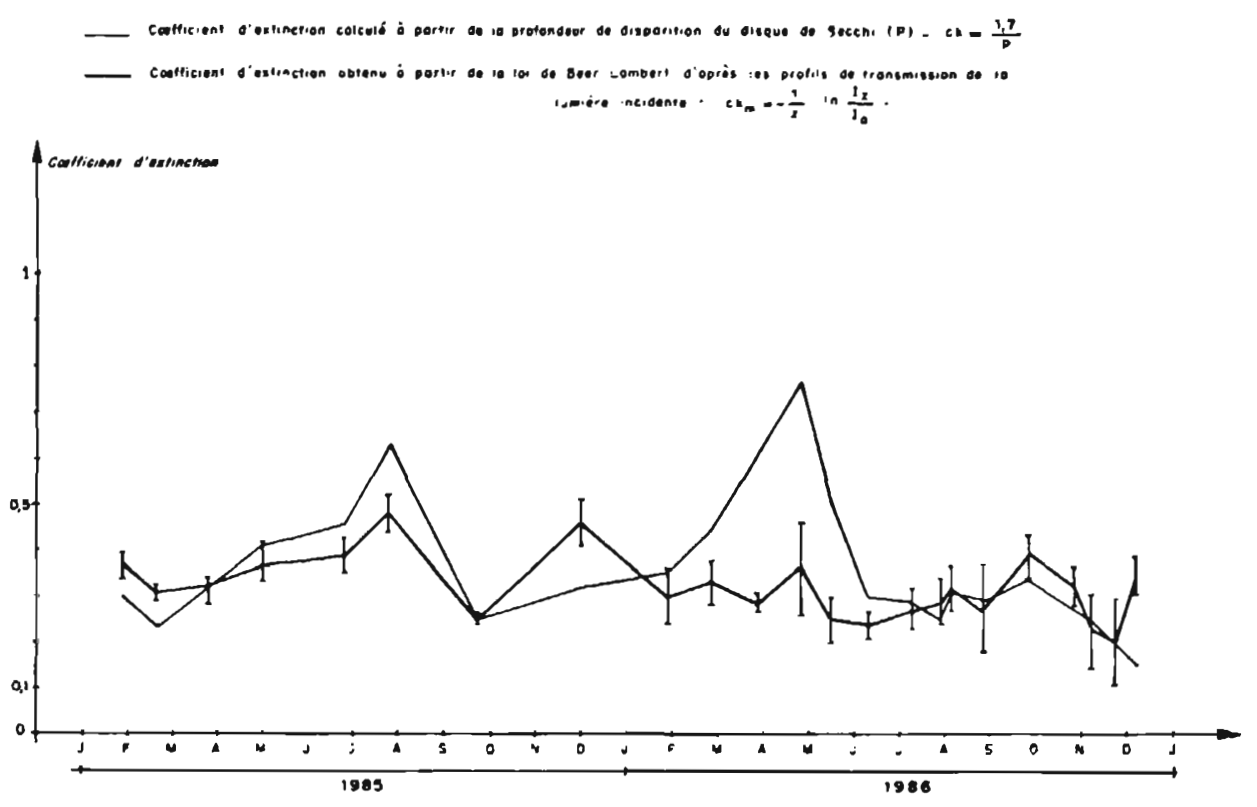

Flg. 5. - Evolution du coefficient d'extinction au cours des années 1985 et 1986.

$44,7 \%$. II existe même une forte corrélation entre IS et $S:$ IS $=-0,148$ $\times S+8,58(r=0,81 ; n=23$, significatif à plus de $1 \%$ ).

Contrairement au quantamètre qui mesure directement l'intensité du rayonnement lumineux à une profondeur donnée, la méthode du disque de Secchi prend en compte la lumière renvoyée par le disque, ce qui augmente l'imprécision de la mesure et pourrait expliquer les fluctuations du pourcentage de lumière transmise is.

En raison de l'incertitude des résultats obtenus avec le disque de Secchi et des très bons ajustements à la loi de Beer Lambert des profils de transmission de lumière déterminés avec la cellule photoélectrique, nous utiliserons les coefficients d'extinction calculés à partir du quantamètre.

Les mesures du coefficient d'extinction sont mensuelles. Les chronologies journalières sont calculées par interpolation linéaire.

Analyse du phytoplancton, du zooplancton et sels nutritifs.

Les prélèvements effectués aux différentes dates d'échantillonnage en 1986, ont permis de décrire la dynamique des populations algales et zooplanctoniques (fig. 6).

Les études de Bin Mole, Pont et Vaquer (1986) et Elhasa (1987) ont montré que le peuplement phytoplanctonique est constitué de 69 uni- 

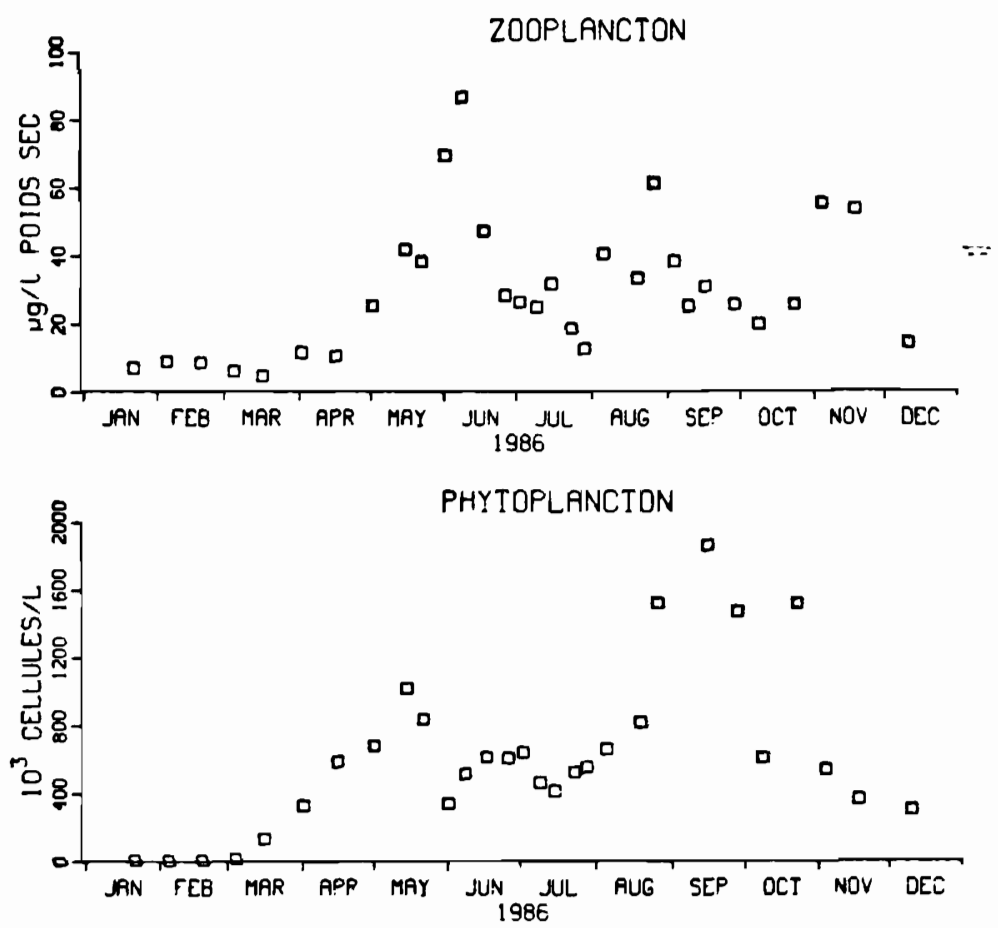

Fig. 6. - Valeur des concentrations du plancton pour 1986.

tés taxonomiques parmi lesquelles 2 groupes dominent:

*des pyrrophytes de petite taille (environ $10 \mu \mathrm{m}$ de diamètre) : ce sont des phytoflagellés du genre Chroomonas, Chromolina, Cryptomonas,

*des diatomées centriques (dont le diamètre est inférieur à $10 \mu \mathrm{m})$ : genre Cyclotella.

Durant la phase hivernale de mélange (décembre 85 à mars 86 ), les concentrations et les biomasses sont très faibles (biomasse $1 \mathrm{mg} / \mathrm{m}^{3}$ ). Un premier pic est observé au printemps, correspondant notamment au déve- loppement rapide d'une population de Chroomonas.

Durant la phase de stratification, en période estivale, on observe 2 poussées (une en août, l'autre fin octobre) séparées par des périodes où les teneurs restent du même ordre qu'en hiver. Ces pics correspondent au développement des diatomées centriques du genre Cyclotella.

Les prélèvements effectués en différents points du lac (fig. 2) ont montré une répartition spatiale assez homogène (fig. 7). 


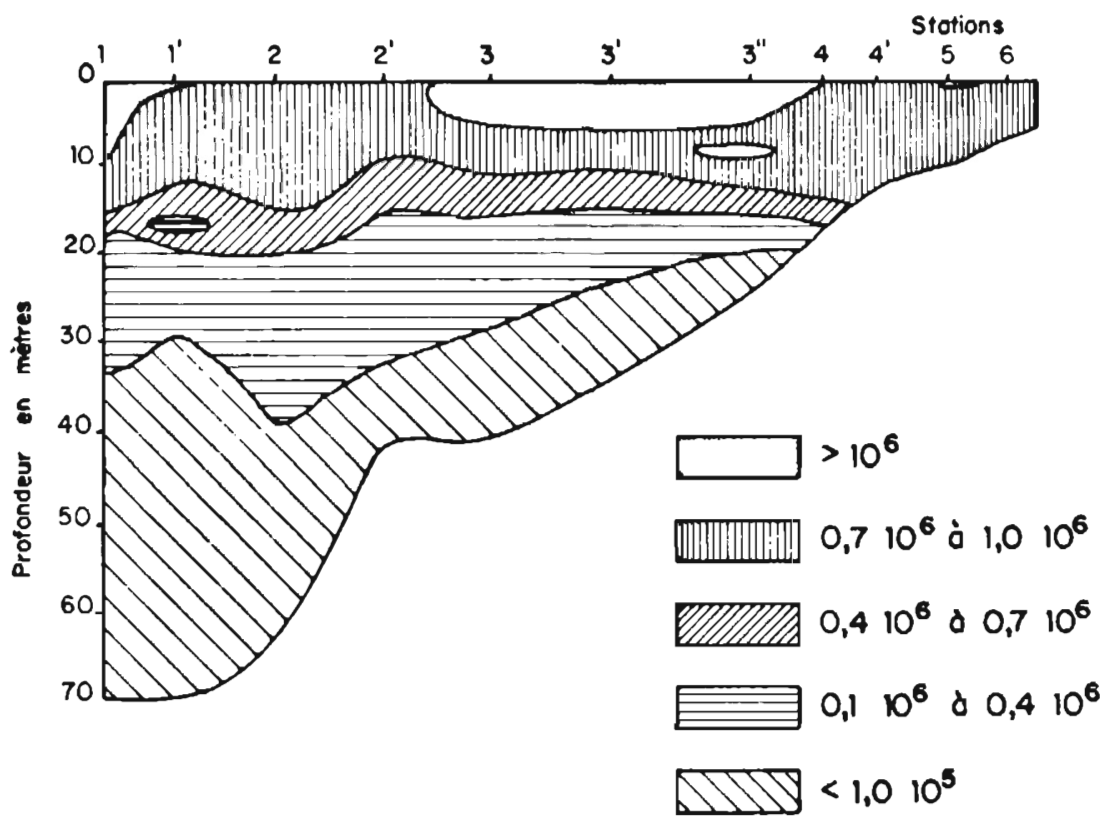

Fig. 7. - Répartition spatiale des densités totales d'algues en nombre d'individus.

Le zooplancton est composé de rotifères (microzooplancton) dont la biomasse ne dépasse pas $0,6 \mathrm{mg}$ de poids $\mathrm{sec} / \mathrm{m}^{3}$, de copépodes et cladocères (macrozooplancton) qui ont une biomasse plus importante (de 7 à $100 \mathrm{mg}$ de poids $\mathrm{sec} / \mathrm{m}^{3}$.

Le zooplancton présente 3 pics aux mêmes périodes que les algues: au printemps, en août puis en novembre. Si le pic de printemps est légèrement décalé par rapport au bloom phytoplanctonique, le phénomène classique "proie-prédateur" est inversé en été, période durant laquelle la dynamique des populations est plus rapide: il est alors possible que la croissance algale soit freinée par la prédation du zooplancton herbivore, lui-même disparaissant rapidement en raison de la prédation importante du niveau trophique supérieur (zooplancton carnivore et alevins).

Cette hypothèse ne peut pas être vérifiée actuellement car nous ne possédons pas de mesures concernant les consommateurs d'ordre supérieur mais d'après les observations faites sur le terrain le nombre de prédateurs du zooplancton herbivore augmente considérablement en période estivale.

\section{Les sels nutritifs.}

Les concentrations en ions $\mathrm{PO}_{4}^{3-}$ sont toujours inférieures à $1 \mu \mathrm{g} / \mathrm{l}$ (quantités trop faibles pour être détectées par les appareils de mesure). Les phosphates, éléments nutritifs limitants, sont recyclés en permanence 
ce qui peut expliquer les faibles valeurs observées au cours de l'année.

\subsection{Choix des variables.}

Le modèle doit être considéré comme un outil de base destiné essentiellement à déterminer l'ensemble des processus fortement impliqués dans le fonctionnement du système: les variables sont donc peu nombreuses.

\section{Variables de forçage.}

- lumière incidente,

- coefficient d'extinction de la lumière,

- température de la couche de surface,

- température de la couche de fond,

- profondeur de la thermocline et volumes respectifs des 2 couches.

\section{Variables d'état.}

Elles ont été choisies en fonction des mesures obtenues sur le terrain:

- 2 groupes d'algues : les phytoflagellés qui se développent au printemps et les diatomées centriques qui apparaissent en été.

- 1 groupe de zooplancton herbivore: le zooplancton est traité comme un seul groupe homogène en considérínt seulement la biomasse totale sans tenir compte des différents stades d'évolution des espèces : les données recueillies sur le terrain ne nous permettent pas une telle distinction : en effet, le zooplancton herbivore est classé uniquement en 2 catégories de taille, le macrozooplancton et le microzooplancton. Ce dernier, composé de rotifères sera né- gligé étant donné sa très faible biomasse.

- le phosphore : Le phosphore est le seul élément nutritif pris en compte car il est limitant pour la croissance phytoplanctonique.

- la matière organique : c'est un compartiment nécessaire au recyclage des éléments organiques provenant de l'excrétion du zooplancton et de la décomposition du plancton mort.

\subsection{Mise en équation des phéno- mènes biologiques.}

Le schéma du fonctionnement du modèle est donné par la figure 8. La mise en équations de l'évolution de ces différentes variables, pour un milieu homogène horizontalement, nous permet de préciser les formes d'interactions entre ces éléments et les différentes hypothèses adoptées pour le modèle.

L'évolution des compartiments dans le modèle peut s'exprimer simplement :

phytoplancton $=f$ (croissance - prédation par le zooplancton). zooplancton $=f$ (croissance - excrétion liquide - mortalité).

phosphore dissous $=f$ (reminéralisation de la matière organique + excrétion liquide du zooplancton pompage du phytoplancton).

matière organique $=f$ (excrétion et mortalité du zooplancton -reminéralisation du phosphore organique). 


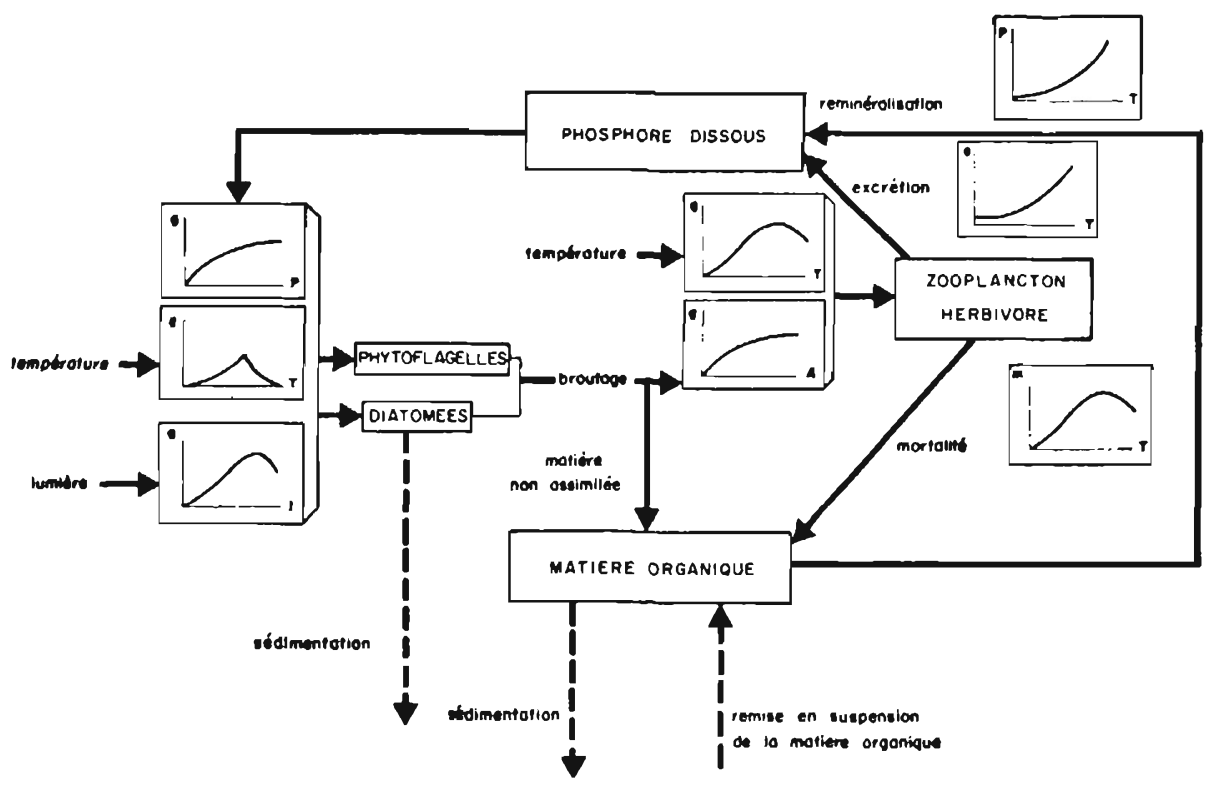

Fig. 8. - Schéma de fonctionnement du modèle.

\section{Évolution du phytoplancton.}

L'évolution du phytoplancton dépend de sa croissance (nutrition et photosynthèse) et de sa disparition (mortalité naturelle et prédation par le zooplancton).

La lumière, la température et la concentration en phosphore sont les paramètres susceptibles de diminuer le taux spécifique de la croissance du phytoplancton lorsqu'ils s'éloignent des valeurs optimales.

L'équation permettant de décrire l'évolution temporelle de la biomasse d'une population d'algues BA s'écrit :

$$
\frac{\mathrm{d} B A}{\mathrm{dt}}=\mu \max \cdot \mathrm{BA}-\text { pred } \cdot \mathrm{BA}
$$

$\mu$ max représente le taux de croissance des algues dans les conditions optimales. Pred représente le taux de prédation exercé par le zooplancton. Le taux de croissance optimal est affecté de facteurs de réduction liés à la température $(F T)$, à la lumière (FLUM) et à la disponibilité en éléments nutritifs, ici le phosphore (FP). On suppose que les 3 facteurs agissent indépendamment sur la croissance algale d'où leur effet multiplicatif :

$$
\frac{d B A}{d t}=\mu \max \cdot F T \cdot F U M \cdot \text { FPBA - pred.BA }
$$

Lorsque 2 espèces d'algues sont prises en compte, il existe des phénomènes complexes de compétition interspécifiques qu'il est difficile de modéliser. Nous différencions donc les phytoflagellés des diatomées par leur optimum de croissance et leurs préférences nutritionnelles. 


\section{Effet de la température.}

L'influence de la température sur le développement algal se traduit de façon classique par une dépendance exponentielle : la température, en accélérant les réactions enzymatiques, augmente la croissance jusqu'à un seuil au-delà duquel elle devient défavorable.

La représentation classique des effets de la température sur le taux de croissance du phytoplancton est donnée par les travaux d'Eppley (1972) sur différentes algues marines et d'eau douce (figs. 9 et 10). Le taux de croissance de chaque espèce de phytoplancton augmente avec la température jusqu'à un optimum puis décroît rapidement.

Pour représenter l'effet de la température sur la croissance du phyto-

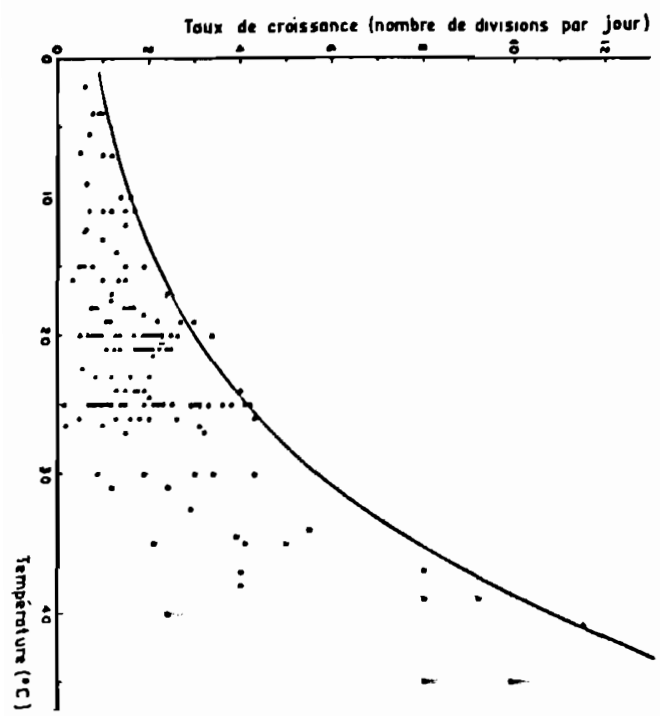

Fig. 9. - Effets de la température sur le taux de croissance du phytoplancton d'après Eppley, 1972. plancton, nous utilisons l'équation de Peeters et Eilers modifiée (Thébault, 1984) et de Jorgensen (Garçon, 1982).

Équation de Peeters et Eilers modifiée.

Elle fait intervenir 3 paramètres dont 2 ont une signification biologique importante : température de croissance nulle (TO), température optimale de croissance (Topt). Le $3^{\mathrm{e}}$ paramètre est un coefficient qui détermine la cambrure de la courbe (b). II détermine l'étendue de la gamme de température sur laquelle les algues ont un taux de croissance élevé.

Soient $T$, la température de l'eau et $\mathrm{FT}$ le facteur de réduction de la

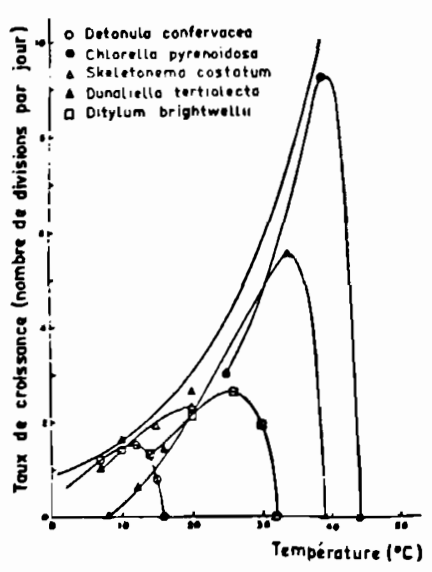

Fig. 10. - Taux de croissance en fonction de la température pour 5 espèces d'algues unicellulaires avec des températures optimales de croissance différentes d'après Eppley, 1972. 
croissance du phytoplancton due à la température :

$X=(T-T O) /(T o p t-T O)$

$F T=2(1+b) \cdot X\left(X^{2}+2 b X+1\right)$

Équation de Jorgensen (1976).

Elle donne une courbe symétrique par rapport à la température optimale de croissance, ce qui permet en particulier de modéliser une chute assez brutale du taux de croissance lorsque la température optimale est dépassée.

$$
F T=\exp \left(-2,3\left|\frac{T-T o p t}{15}\right|\right)
$$

Dans cette équation, la température de croissance nulle n'intervient pas.

\section{Effet de la lumière.}

La fonction de limitation par la lumière introduit une relation existant entre la photosynthèse et l'éclairement.

L'équation de Steele (1962) a été largement utilisée par les modélisateurs (Kremer et Nixon 1978, Wang et Halerman 1982) :

$$
\text { FLUM }=\frac{1}{\text { lopt }} e^{\left(1-\frac{1}{\text { lopt }}\right)}
$$

FLUM : rapport entre la productivité à une profondeur donnée et la productivité maximale.

lopt : intensité optimale de production algale.

1 : intensité à une profondeur donnée.

En intégrant sur la verticale la loi de Steele, on obtient:

$$
\begin{aligned}
\text { FLUM } & =\int_{0}^{H} \frac{1}{\text { opt }}\left(e^{1-1 / \text { lopt }}\right) \\
& =\frac{e}{K H} \cdot\left(\exp \left(-\frac{\mathrm{lZ}}{\text { lopt }}\right)-\exp \left(-\frac{10}{\text { lopt }}\right)\right)
\end{aligned}
$$

$\mathrm{K}$ : coefficient d'extinction de la lumière

$\mathrm{H}$ : profondeur de la thermocline (en m)

$\mathrm{IZ}$ : intensité de la lumière à la hauteur $\mathrm{H}$

lo : intensité de la lumière à la surface.

avec $\mathrm{IZ}=10 e^{\mathrm{KH}}$ (équation de BeerLambert).

L'équation de Steele ne donne qu'une approche imparfaite du phénomène d'inhibition par la lumière (Dauta, 1983), pour cette raison, de même que Thébault (1984), nous utiliserons l'équation de Peeters et Eilers construite sur une base biochimique dont les ajustements sur des données expérimentales sont excellents.

FLUM $=2(1+b) \frac{1 / \text { lopt }}{(1 / \text { lopt })^{2}+2 b(1 / \text { lopt })+1}$

où $b$ est un coefficient d'acuité de la courbe.

En intégrant entre la surface et la profondeur de la thermocline l'équation devient :

$$
\begin{aligned}
& \text { FLUM }=\frac{2}{H \cdot K}\left(\frac{1+b}{\sqrt{1-b^{2}}}\right) \\
& .\left(\operatorname{Arctg}\left(\frac{10+b \cdot \text { lopt }}{\text { lopt. } \sqrt{1-b^{2}}}\right)-\operatorname{Arctg}\left(\frac{\text { l } z+b \cdot \operatorname{lopt}}{\text { lopt } \sqrt{1-b^{2}}}\right)\right)
\end{aligned}
$$

Dans une première étape, nous supposons que les 3 facteurs agissant sur la croissance du phytoplancton sont indépendants, mais il existe une interaction entre la lumière et la température comme l'a montré Dauta (1983) par ses travaux sur 8 espèces d'algues : l'intensité optimale de production algale est plus faible lorsque la température diminue. Pour se développer à basse température le phy- 
toplancton requiert moins de lumière (figure 11).

\section{Effet des nutriments.}

La littérature offre de multiples exemples de limitation de la croissance des algues par les éléments nutritifs. Lorsque la population phytoplanctonique n'est pas limitée par le nutriment, sa croissance tend vers un optimum qui dépend uniquement des conditions de température et de lumière.

L'équation de Monod (1949) est souvent utilisée pour décrire la relation existant entre la croissance et la concentration du nutriment limitant (ici, le phosphore), par une fonction hyperbolique :

$$
\mathrm{FP}=\frac{\mathrm{PO} 4}{\mathrm{KS}+\mathrm{PO} 4}
$$

où KS est la constante de demi-saturation en substrat et $\mathrm{PO} 4$ la concentration en ion $\mathrm{PO}_{4}^{3}$.
La figure 12 représente la réponse du taux de croissance à l'élément limitant pour 2 espèces d'algues ayant des constantes de demi-saturation différentes.

L'hypothèse de base dans l'équation de Monod réside dans l'existence d'un raport constant entre la biomasse métabolisée et le substrat externe consommé.

La croissance du phytoplancton est considérée comme un processus à une seule étape : l'absorption du sel nutritif entraîne directement la croissance du phytoplancton.

Le modèle proposé ne tient pas compte du phénomène de consommation de luxe (stockage intercellulaire des éléments nutritifs). D'après Wang et Harleman (1982), la prise en compte des processus internes d'absorption du phosphore n'apporteront

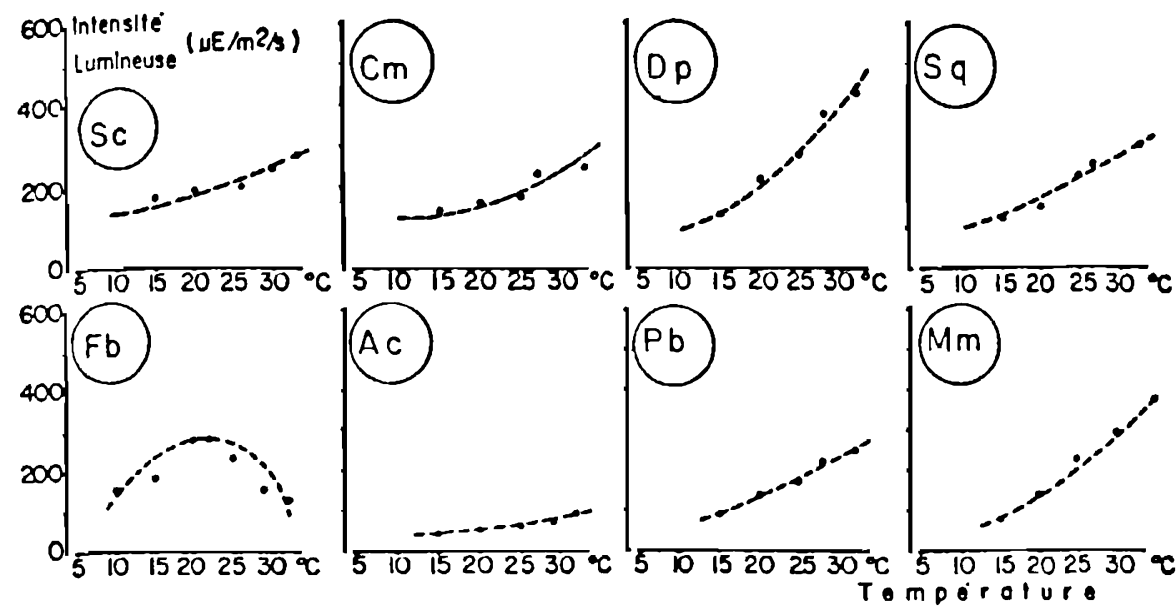

Fig. 11. - Variation de lopt en fonction de la température pour 8 espèces d'algues unicellulaires (Scenedesmus crassus, Coelastrum microsporum, Dictyosphaerum pulchellum, Scenedesmus quadricauda, Fragilaria bidens, Anaeba cylindrica, Pediastrum boryanum, Monoraphidium minutum). D'après Dauta (1983). 

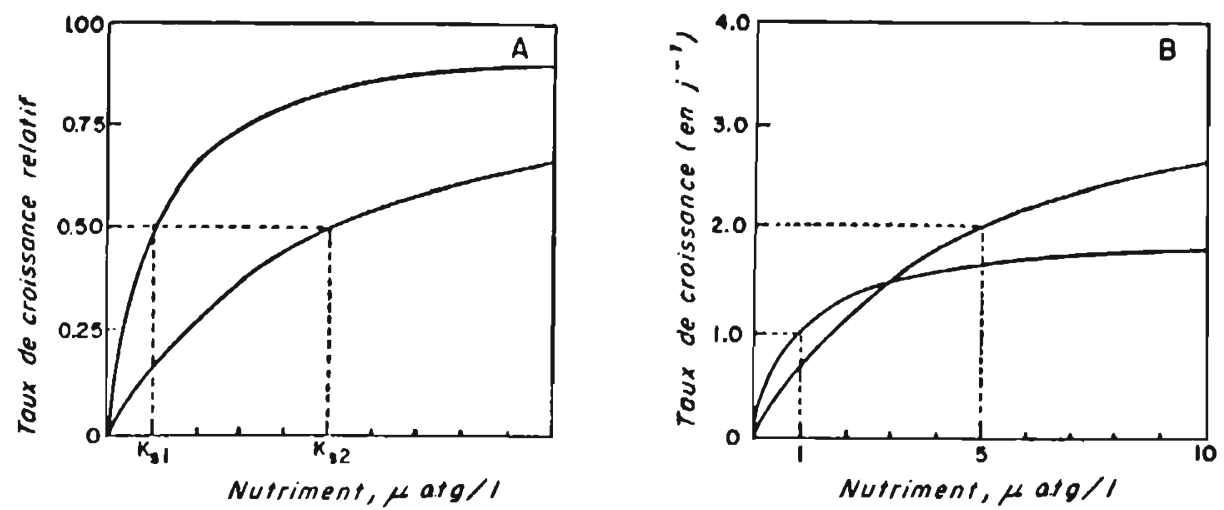

Fig. 12. - Taux de croissance de 2 espèces de phytoplancton (ayant des constantes de demi-saturation différentes) en fonction de la concentration en sel nutritif (Kremer et Nixon, 1978).

des différences par rapport au modèle de base interne d'absorption de la cinétique de Monod que pendant de très courtes phases, entraînant, de plus, de nombreux paramètres supplémentaires.

\section{Mortalité du phytoplancton.}

Nous ne prenons en compte que la prédation par le zooplancton herbivore.

II est difficile de déterminer les contributions respectives des facteurs dont dépend la mortalité naturelle: température extrêmement basse ou élevée, concentration en sels nutritifs trop faibles. Nous ferons l'hypothèse que la mortalité naturelle est négligeable par rapport à la prédation. Toutefois, nous avons introduit un coefficient de mortalité des diatomées dans le sédiment.

\section{Évolution du zooplancton.}

Comme dans la plupart des modèles, la croissance du zooplancton est exprimée comme le produit de deux fac- teurs dont l'un dépend de la température (ZT) et l'autre de la quantité de phytoplancton (ZM).

Le facteur limitant la croissance du zooplancton est une fonction exponentielle de la température représentée par l'équation de Peeters et Eilers modifiée ou de Jorgensen, relations déjà utilisées pour simuler l'effet de la température sur le taux maximum de croissance algale.

Parmi les équations représentant la fonction de broutage, deux expressions sont utilisées: IVLEV et MICHAELIS-MENTEN.

Loi d'IVLEV (1966)

$Z M=1-\exp (-$ R.BTOT)

MICHAELIS-MENTEN

$\mathrm{ZM}=\mathrm{BTOT} /(\mathrm{BTOT}+\mathrm{KN})$.

avec :

$\mathrm{R}=$ constante contrôlant le degré de limitation de l'ingestion par la concentration en nourriture.

BTOT = concentration totale en nourriture affectée au zooplancton $\mathrm{KN}=$ constante de demi-saturation. 
Dans ces modèles, l'efficacité d'assimilation des algues est une constante. Les versions modifiées incluent le fait qu'au-dessous d'une certaine concentration donnée de nourriture, les animaux cessent de se nourrir (Garçon, 1982). Nous admettons ici, que la valeur seuil est de l'ordre de $5.10^{4}$ cellules/l.

II est possible d'utiliser la loi d'IVLEV lorsque le compartiment phytoplanctonique ne comprend qu'une espèce d'algue. Par contre, en présence de 2 groupes d'algues, diatomées et les phytoflagellés, nous considérons que le broutage s'opère de façon sélective.

L'expression est divisée en un terme de type Michaelis-Menten reliant le broutage à la concentration totale en nourriture et un autre terme représentant la fraction de nourriture totale ingérée provenant d'une certaine catégorie de proie. Nous ferons l'hypothèse que les diatomées qui présentent une frustule de silice sont ingérées moins facilement que les phytoflagellés.

L'équation de Michaelis-Menten devient :

$$
\begin{aligned}
Z N & =(w 1 . \text { Phyflag }+w 2 . \text { diat }) \\
& /(K N+w 1 . \text { Phyflag }+w 2 . \text { diat })
\end{aligned}
$$

avec :

$\mathrm{KN}$ : constante de demi-saturation. $w_{1}$ et $w_{2}$ : coefficients de proportionnalité représentant la fraction de nourriture ingérée.

Phyflag et diat : les concentrations en phytoflagellés et en diatomées.
ZN flagellés $=\frac{w_{1} \cdot \text { Phyflag }}{K N+w_{1} \cdot \text { Phyflag }+w_{2} \cdot \text { diat }}$ $=$ prédation du zooplancton sur les phytoflagellés

$\mathrm{ZN}$ diatomées $=\frac{\mathrm{w}_{2} \cdot \text { diat }}{\mathrm{KN}+\mathrm{w}_{1} \cdot \text { Phyflag }+\mathrm{w}_{2} \cdot \text { diat }}$ $=$ prédation du zooplancton sur les diatomées.

Si nous différencions la sélection du phytoplancton, nous estimons par contre que le taux d'assimilation des algues ingérées est constant. La nourriture ingérée par le zooplancton mais non assimilée est perdue par excrétion solide sous forme de pelotes fécales et alimente le compartiment matière organique.

\section{Disparition du zooplancton.}

L'excrétion liquide du zooplancton est une fonction exponentielle de la température (Garçon, 1980).

$$
\text { EXCR }=a e^{b T}
$$

EXCR = taux d'excrétion

$a, b=$ coefficients

$\mathrm{T}=$ température de l'eau.

La mortalité des animaux est due principalement à la prédation du niveau trophique supérieur, généralement construite de façon identique au terme de broutage. Comme nous ne simulons que les herbivores, nous supposerons dans un premier temps que le taux de mortalité est constant. Dans un deuxième temps, afin de reproduire l'augmentation de la prédation pendant la période estivale, nous simulerons la mortalité du zooplancton herbivore par une fonction dépendant de la température (fonction d'Eilers). 
Évolution du phosphore et de la matière organique.

Le phosphore, sous la forme d'ion $\mathrm{PO}_{4}^{3-}$ est absorbé directement par les algues. II provient de l'excrétion liquide du zooplancton et de la reminéralisation de la matière organique, composée de pelotes fécales et d'animaux morts. La reminéralisation, processus biologique, dépend de la température.

Nous utilisons la fonction proposée par Kremer et Nixon (1978):

PO4 $=\mathrm{a} e^{\mathrm{bT}}$

$\mathrm{a}, \mathrm{b}$ : coefficients

$\mathrm{T}$ : température de l'eau en ${ }^{\circ} \mathrm{C}$.

\section{Aspects numériques.}

L'échelle de temps des phénomènes reproduits est d'une journée. Le système d'équations différentielles constituant le modèle est résolu pour chaque couche par la méthode de Runge Kutta d'ordre 4 à pas constant. Le pas d'intégration est de 3 heures (il a été choisi après une étude de sensibilité). Les échanges entre les couches sont calculés par différence finie à l'échelle journalière. Toutes les 24 heures, les concentrations des variables d'état sont calculées en tenant compte de 4 processus :

- dilution des 2 masses d'eau qui dépend des variations de la profondeur de la thermocline,

- sédimentation des diatomées et de la matière organique,
- remise en suspension de la matière organique du sédiment lorsque la couche de surface atteint le fond,

- répartition verticale du zooplancton: l'échelle de temps choisie étant d'un jour, nous admettons que la probabilité de trouver les animaux dans l'une ou l'autre couche est identique.

Le bilan de masse, exprimé en phosphore, est régulièrement calculé au cours de la simulation afin de contrôler la conservativité du modèle.

Le calcul débute le $1^{\text {er }}$ janvier 1985 bien que nous ne disposions pour l'instant d'aucune mesure pour l'année 1985. Cette première année de simulation permet d'assurer la convergence du modèle et d'effacer la mémoire de l'état initial.

Pour les variables du modèle qui sont mesurées en 1986 (phytoplancton, zooplancton, phosphore), les valeurs initiales choisies sont celles de janvier 1986.

Pour celles qui ne sont pas mesurées (matière organique dans le sédiment et dans l'eau, algues dans le sédiment), nous avons procédé par itérations successives en supposant qu'en hiver l'écosystème repasse par un état qui varie peu d'une année sur l'autre. A partir de valeurs initiales nulles pour ces variables, nous avons considéré les valeurs obtenues après un an de simulation. Ces valeurs ont été introduites comme nouvelles valeurs initiales jusqu'à convergence du processus, obtenue après 3 itérations. 
Le système d'équations et la schématisation du programme sont les suivants :

\section{Notations utilisées}

\begin{tabular}{|c|c|c|}
\hline Variables & Notations & Unités \\
\hline $\begin{array}{l}\text { Phytoplancton: } \\
\text { Flagellés } \\
\text { Phytoplancton : } \\
\text { Diatomées } \\
\text { Zooplancton } \\
\text { Phosphore } \\
\text { Matiere organique. }\end{array}$ & $\begin{array}{l}\text { Flag. } \\
\text { Diat. } \\
\text { Zoo. } \\
\text { Phos. } \\
\text { MO. }\end{array}$ & $\begin{array}{l}10^{9} \text { cellules/I } \\
10^{9} \text { cellules/I } \\
\mu g \text { poids sed/I } \\
\mu \text { atg } P / I \\
\mu \text { atg } P / I\end{array}$ \\
\hline
\end{tabular}

\section{ABRÉVIATIONS}

$T$ = température de l'eau.

$Z T$ = facteur de réduction de la mortalité du zooplancton due à la température.

FT = facteur de réduction de la croissance du phytoplancton due à la température.
$\mathrm{FTZ}$ = facteur de réduction de la croissance du zooplancton due à la température.

FLUM = facteur de réduction de la croissance du phytoplancton due à la lumière.

$\mathrm{FN}=$ facteur de réduction de la croissance du phytoplancton due à la concentration en nutriment.

assim. : taux d'assimilation de la nourriture ingérée par le zooplancton.

W1, W2 : coefficients représentant la sélectivité du zooplancton par rapport aux 2 catégories d'algues.

CP1, CP2 : rapport Phosphore/Biomasse en $\mu$ atg $P / 10^{9}$ cellules.

$\mathrm{Kn}$ : constante de demi-saturation en $\mu$ atg $P / l$.

$a, b, \alpha, \beta$ : coefficients sans dimension.

$\mu \max _{1}, \mu \max _{2}$ : taux de croissance des phytoflagellés et des diatomées dans les conditions optimales.

Modélisation numérique : système d'équations du modèle.

Zooplancton herbivore

$$
\begin{aligned}
& \frac{\mathrm{dZoo}}{\mathrm{dt}}=\left[\left(\frac{\mathrm{wl} \cdot \text { Flag }}{\text { Algues }} \cdot \mathrm{cp}_{1} \cdot \mathrm{assim} \cdot\right) \cdot \mathrm{FTZ}+\left(\frac{\mathrm{W} 2 \cdot \mathrm{diat}}{\text { Algues }} \cdot \mathrm{cp}_{2} \cdot \operatorname{assim}\right) \cdot \mathrm{FTZ}-\mathrm{ae} \mathrm{e}^{\mathrm{bT}}-\mathrm{ZT}\right] \cdot \mathrm{Z} \infty \\
& \text { croissance }
\end{aligned}
$$

\section{Diatomées}

Phytoflagellés

$$
\begin{aligned}
& \frac{d \text { diat }}{d t}=\left(\mu \max _{2} \cdot F T \cdot F L U M \cdot F N\right) \cdot d i a t-\left(\frac{w 2 \cdot d i a t}{\text { Algues }} \cdot Z 00\right) \\
& \text { production primaire }
\end{aligned}
$$

$$
\begin{aligned}
& \frac{\mathrm{d} \text { Flag }}{\mathrm{dt}}=\left(\mu \max _{1} \cdot \mathrm{FT} \cdot \mathrm{FLUM} \cdot \mathrm{FN}\right) \cdot \text { flag }-\left(\frac{\mathrm{w} 1 \cdot \text { Flag }}{\text { Algues }} \cdot \mathrm{Zoo}\right) \\
& \text { production primaire } \\
& \text { broutage }
\end{aligned}
$$


Phosphore

$\left.\frac{d \text { Phos }}{d t}=\left(\alpha \theta^{\beta T} \cdot M O\right)+{ }^{\prime} a e^{b T} \cdot Z O O\right)$

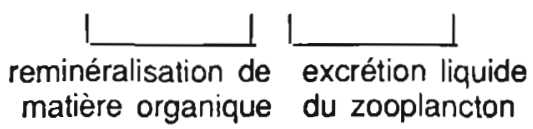

$-\left[\left(\mu \max _{1} \cdot \mathrm{FT} \cdot \mathrm{FLUM} \cdot \mathrm{FN}\right) \cdot \mathrm{Flag} \cdot \mathrm{cp}_{1}+\left(\mu \max _{2} \cdot \mathrm{FT} \cdot \mathrm{FLUM} \cdot \mathrm{FN}\right) \cdot \mathrm{diat} \cdot \mathrm{cp_{2 }}\right]$

pompage du phytoplancton

Matière organique

$$
\begin{aligned}
& \frac{d M O}{d t}=\left[\frac{W 1 \cdot F l a g}{\text { Algues }} \cdot c p_{1}+\frac{W 2 \cdot d i a t}{\text { Algues }} \cdot c p_{2}\right](1-\text { assim }) \cdot Z O O+(Z T \cdot Z O O)-\left(\alpha \mathrm{e}^{\beta T} \cdot M O\right) \\
& \text { phosphore non assimilé par le zooplancton mortalité du } \\
& \text { zooplancton }
\end{aligned}
$$

avec Algues $=\mathrm{KN}+\mathbf{w}_{1} \cdot \mathbf{F l a g}+\mathbf{w}_{2} \cdot$ diat.

\section{3 - RÉSULTATS}

\section{1. Étude de la réponse du modèle au forçage.}

Dans les conditions non limitantes et constantes du milieu, l'évolution d'une proie (représentée ici par le phytoplancton) et de son prédateur (le zooplancton) atteint un état d'équilibre caractérisé par une succession de pics d'amplitude constante.

Afin d'évaluer l'influence des variables de forçage sur l'évolution du plancton, nous simulons dans un premier temps un système proie-prédateur. Les valeurs des paramètres intervenant dans le modèle sont issues de la littérature (Jorgensen,
1979, Waterloopkunding Laboratorium, 1979).

Les hypothèses du modèle sont les suivantes:

- La structure thermique du lac n'évolue pas: la retenue est composée d'une couche de mélange de profondeur constante $(82 \mathrm{~m})$.

- Les valeurs des 3 variables de forçage sont non limitantes: température de l'eau : $14^{\circ} \mathrm{C}$ intensité lumineuse à la surface de l'eau : $350 \mathrm{~W} / \mathrm{m}^{2}$.

coefficient d'extinction de la lumière : $0.15 \mathrm{~m}^{-1}$

- La concentration en nutriments est fixée à 0.03 atg $\mathrm{P} /$.

Dans ces conditions, les facteurs de réduction liés à la température $(F T)$ et à la lumière (FLUM) qui af- 
Schématlsation du programe

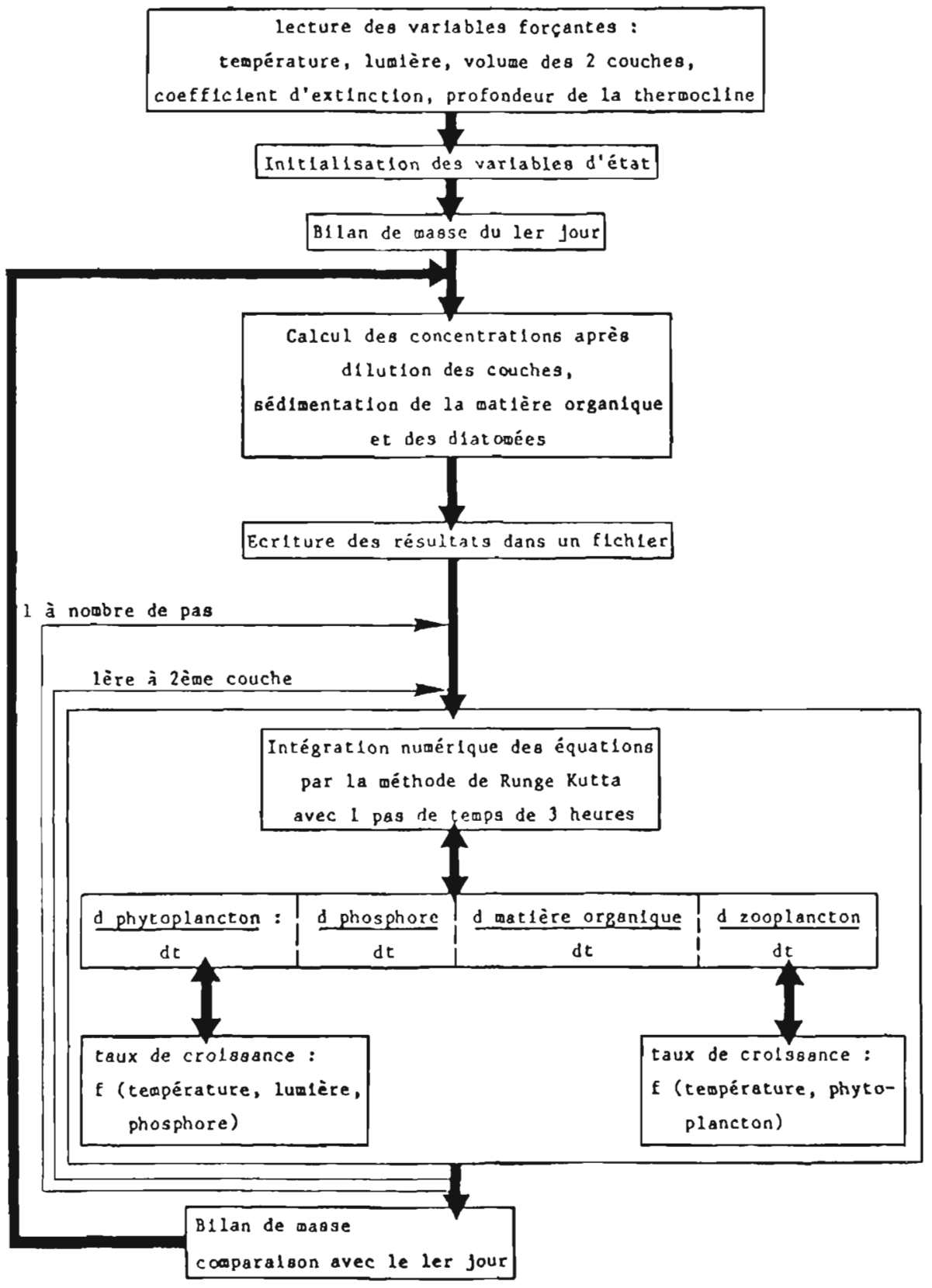


fectent le taux de croissance du plancton, restent constants :

pour le phytoplancton, $\mathrm{FT}=\mathbf{0 . 7 9}$ et $\mathrm{FLUM}=0.18$

pour le zooplancton, $\mathrm{FT}=0.78$

Un système oscillatoire classique est obtenu en 4 mois (fig. 13a). Chaque cycle a une durée de 2 mois et demi. Le pic du zooplancton se produit 13 jours après celui du phytoplancton.

Pour évaluer le rôle de chaque variable sur l'équilibre du système, nous maintenons constantes 2 variables de forçage, la $3^{\mathrm{e}}$ prenant les valeurs mesurées sur le site de Sainte-Croix au cours des années 1985 et 1986. Les résultats sont donnés par la figure 13.

\section{Influence de la luminosité.}

L'intensité du rayonnement solaire intervient seulement sur la photosynthèse. Sa limitation sur la croissance des algues est représentée par la fonction de Peeters et Eilers qui prend aussi en compte le coefficient d'extinction de la lumière $\mathrm{CK}$ et la profondeur de la couche de surface $P$ :

$$
\mathrm{FLUM}=\frac{1}{\mathrm{CK} . \mathrm{P}} \cdot(\mathrm{a}(\mathrm{I}))
$$

où $a(I)$ est une fonction complexe dans laquelle intervient l'intensité lumineuse en surface et à la profondeur $P$.

L'intensité incidente varie de 50 à $350 \mathrm{~W} / \mathrm{m}^{2}$ au cours des années 1985 et 1986. Cependant, comme CK et $P$ restent constants, FLUM varie peu (de 0.12 à 0.18 ) malgré les fortes variations du rayonnement solaire.
Le premier pic est retardé de 15 jours : il apparaît début mars mais l'amplitude des oscillations est très peu modifiée (fig. 13b). On note une légère diminution des valeurs maximales en fin d'automne. La durée des cycles est plus longue en hiver. En laissant constants le coefficient d'extinction et la profondeur de la couche, nous réduisons l'influence du rayonnement solaire.

\section{Influence du coefficient d'extinction} de la lumière.

Les valeurs calculées varient de 0.23 à $0.46 \mathrm{~m}^{-1}$, ce qui entraîne des fluctuations de FLUM comprises entre 0.12 et 0.06 .

La perturbation du système proieprédateur est nettement plus importante: la durée des cycles est plus longue (3 mois) et l'amplitude des pics peut doubler selon la saison (fig. 13c).

\section{Influence de la température.}

La température intervient non seulement sur la croissance du plancton mais aussi sur la reminéralisation de la matière organique, l'excrétion et la mortalité du zooplancton. Les valeurs mesurées sur le site de Sainte-Croix varient de 6 à $23^{\circ} \mathrm{C}$, selon la saison, entraînant de fortes variations du taux de croissance du plancton le facteur de réduction FT varie de 0.17 à 1 pour le phytoplancton et de 0.04 à 1 pour le zooplancton.

Le système proie-prédateur est donc fortement influencé par les variations saisonnières de la tempéra- 


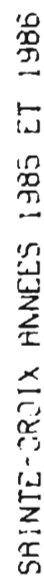
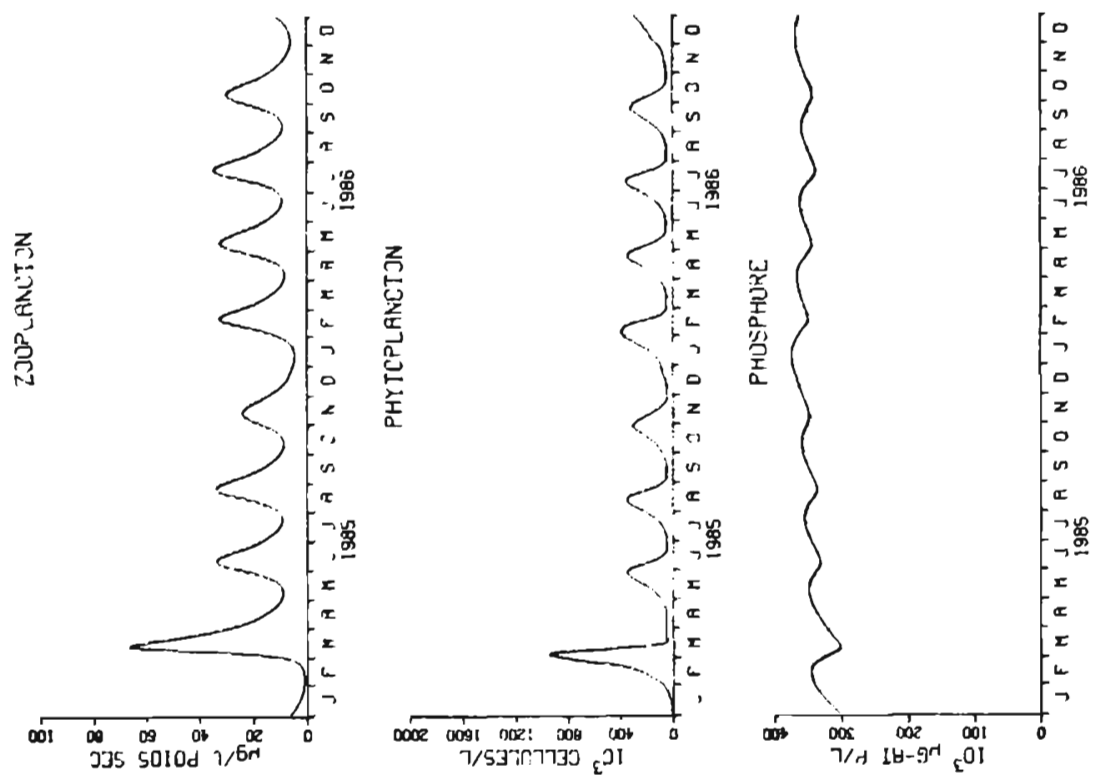

है

点
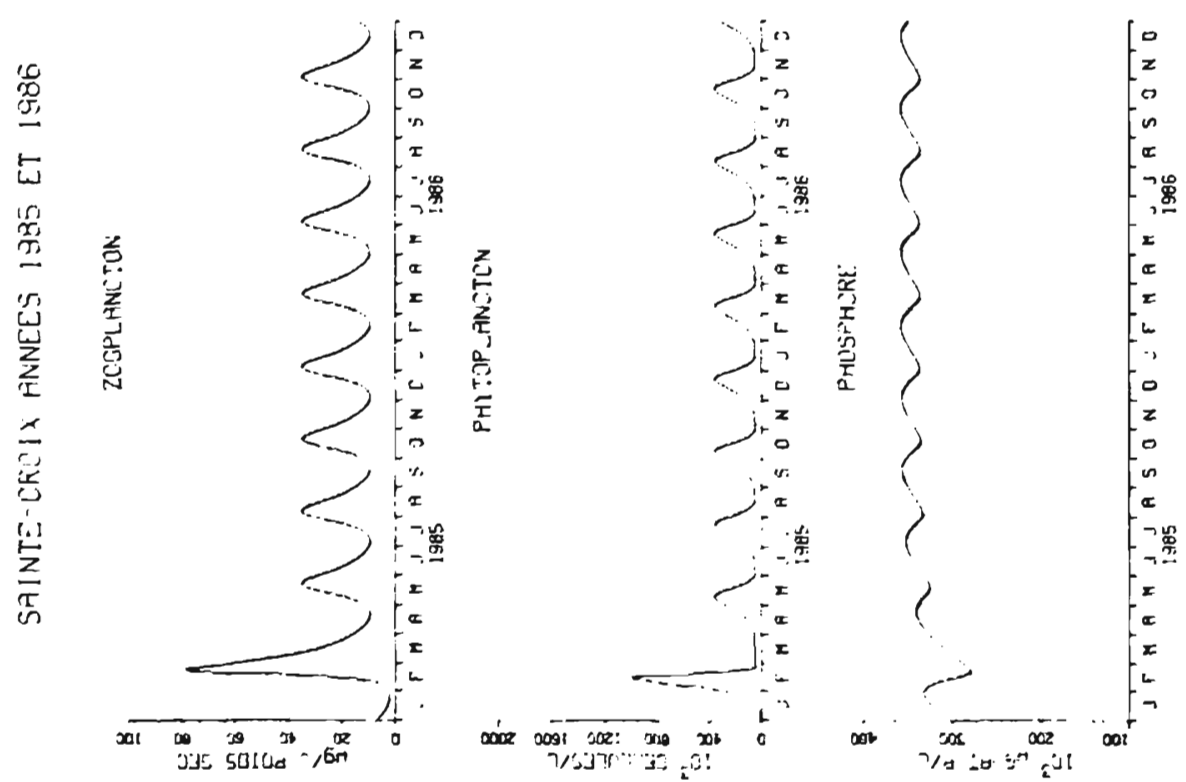

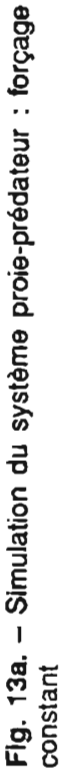


ture de l'eau. Les pics, irréguliers, présentent une amplitude très élevée (fig. 13d).

Effet de la structure bicouche du modèle sur l'évolution de l'écosystème.

Nous avons suivi le comportement des variables d'état en supposant la profondeur de la couche de surface constante, or, la température intervient aussi sur la structure du modèle puisque ses variations déterminent l'apparition de la thermocline. Nous évaluons donc les modifications apportées par la structure bicouche en comparant les concentrations du plancton évoluant dans une ou deux couches (fig. 14).

Quel que soit le nombre de couches considérées, seules les concentrations des variables de la couche de surface sont représentées.

une couche: le forçage correspond aux valeurs mesurées sur le site de Sainte-Croix mais nous considérons constante la profondeur de la couche. Bien que le phosphore ne soit jamais limitant, le nombre de pics est restreint. Les pics qui apparaissent au printemps et en automne résultent des variations saisonnières des variables de forçage (fig. 14a).

deux couches: La profondeur de la couche de mélange est déterminée par la hauteur de la thermocline. La principale différence observée est due à la limitation de la croissance phytoplanctonique par le phosphore: au printemps, le développement des algues est limité rapidement par la disparition du phosphore dans la couche de surface. Au mois d'août, la thermocline s'enfonce de quelques mètres entraînant dans la couche de surface un apport de phosphore provenant de la couche de fond (fig. 14b).

Le système proie-prédateur est donc fortement influencé par la structure verticale de la masse d'eau.

Effet de la mortalité du prédateur sur l'évolution de l'écosystème.

Comme nous l'avons vu précédemment, la croissance du phytoplancton dépend principalement de la température de l'eau et de la concentration en zooplancton (système proie-prédateur). II nous paraît intéressant d'étudier l'influence de la mortalité du zooplancton qui est une fonction de forçage du modèle.

Pour représenter la prédation du niveau trophique supérieur (qui entre dans le terme de disparition du zooplancton), nous étudierons plusieurs possibilités :

- coefficient de mortalité constant et faible: $m=0.02$

- coefficient de mortalité constant et élevé : $m=0.15$.

- coefficient de mortalité variable : pour représenter le phénomène de forte prédation, observé en été, nous relions la mortalité à la température de l'eau par une fonction de forçage (fonction d'Eilers). Les valeurs des paramètres intervenant dans la fonction sont ajustées de façon à ce que la mortalité soit faible en été et forte en hiver. 

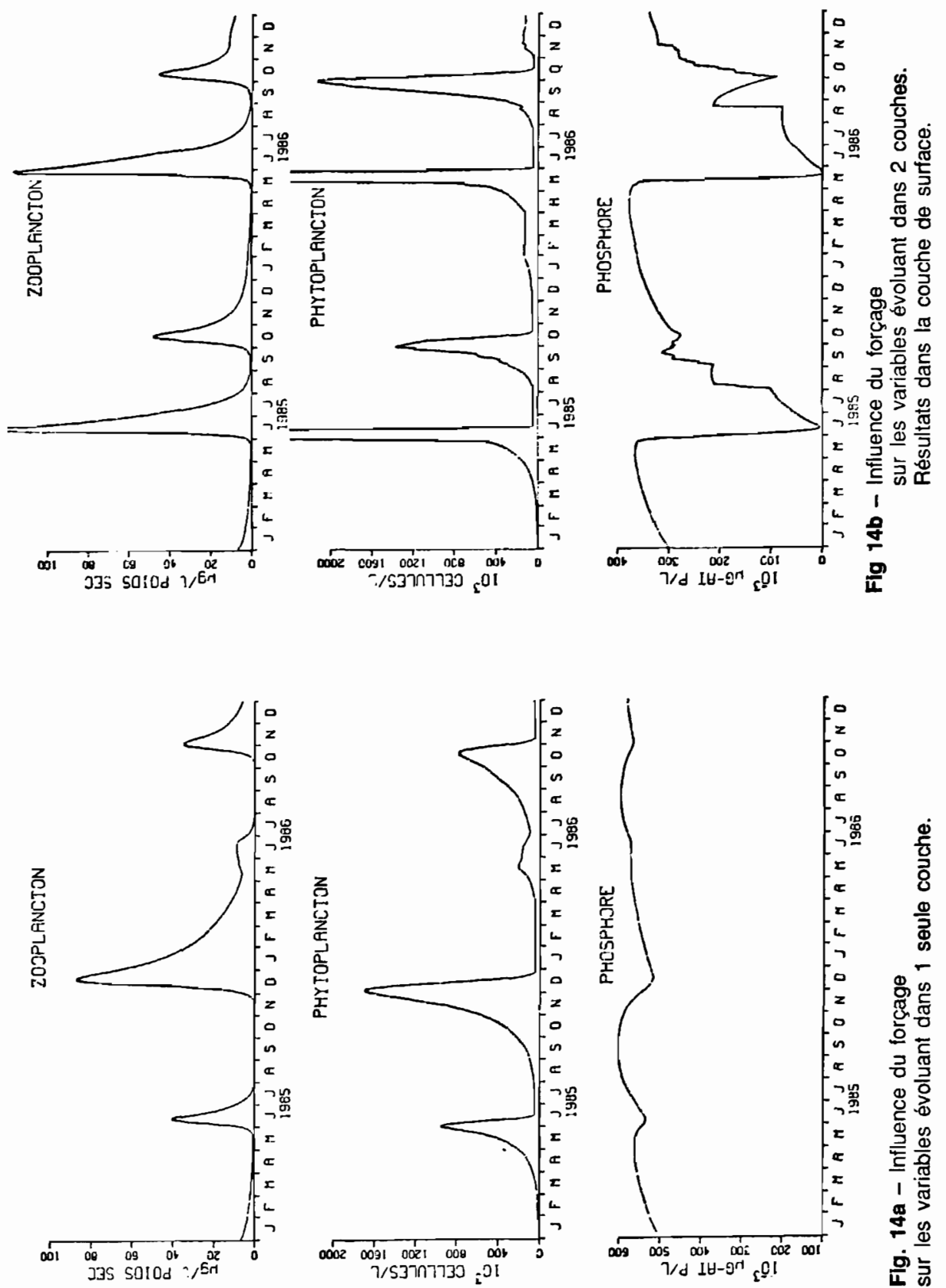
A.-M. Pourcher, M.-J. Salençon
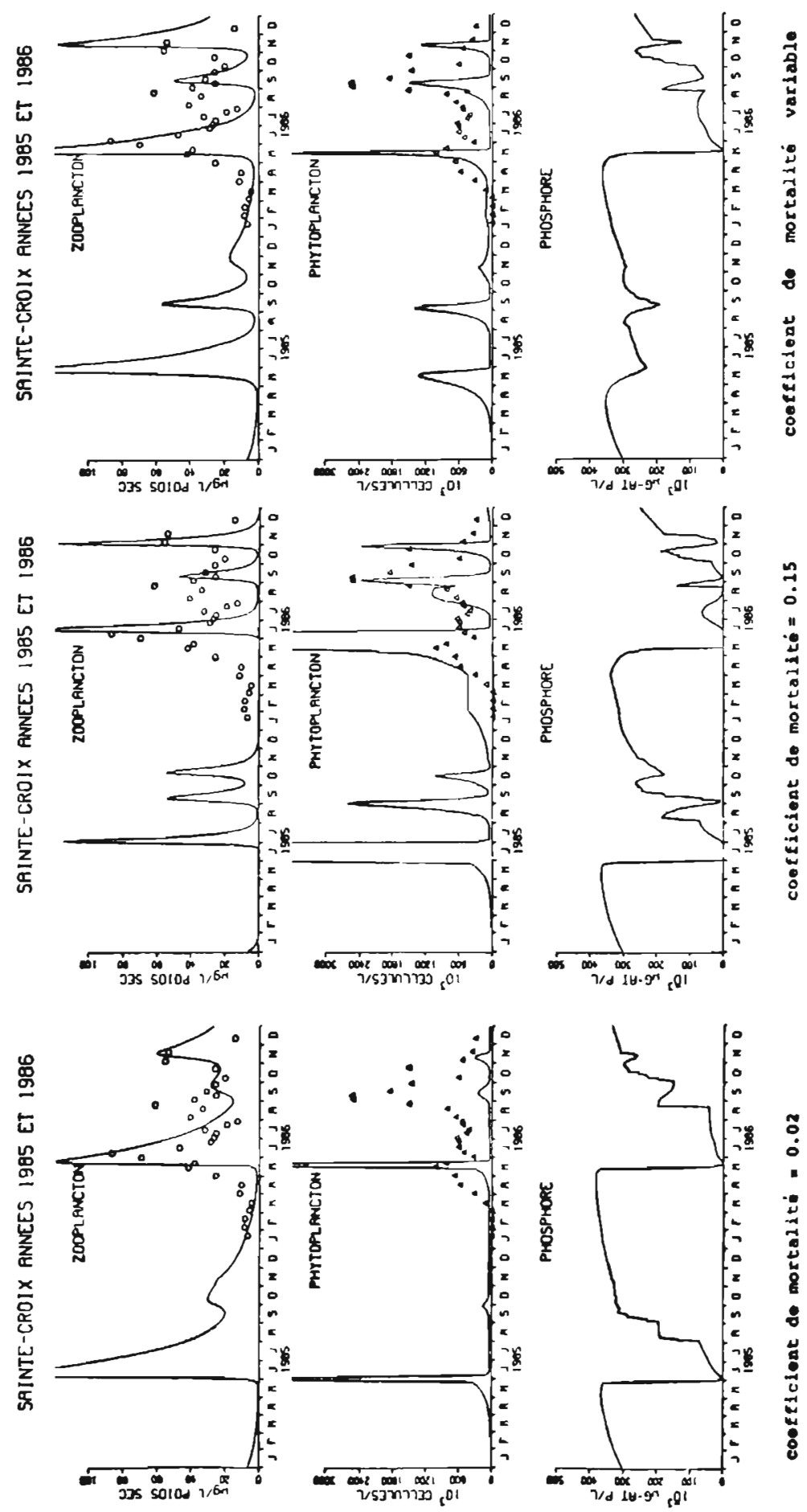
Les résultats (fig. 15) montrent qu'avec un coefficient de mortalité fixé à 0.02 , le zooplancton se développe très rapidement et conserve une biomasse élevée en été, limitant ainsi la croissance des algues. Au contraire, lorsque le coefficient est plus élevé, on note une disparition du zooplancton en hiver et une concentration entre deux pics successifs quasiment nulle.

Les résultats obtenus avec une mortalité dépendant de la température de l'eau nous paraissent plus satisfaisants: le zooplancton est présent à faible concentration en hiver tout en ayant une dynamique de disparition rapide en été.

\subsection{Choix des catégories du phytoplancton.}

II ne nous a pas été possible de représenter le phytoplancton par un seul groupe d'algues. En effet, l'étude de l'influence de la température optimale de croissance du phytoplancton (Pourcher et Salençon - 1988) montre qu'avec une espèce eurytherme, supportant une large gamme de températures, il n'est pas possible de bien représenter les 3 pics du phytoplancton observés sur le site de Sainte-Croix en 1986. De même avec une espèce sténotherme, se développant sur une gamme de températures réduite, le pic de printemps est bien représenté (notamment pour une température optimale de croissance de $12.5^{\circ} \mathrm{C}$ ) mais le développement des algues en été reste limité. Les prélèvements réalisés sur le site ont mon- tré la dominance de phytoflagellés au printemps et de diatomées en été.

II nous paraît donc intéressant d'ajouter un compartiment phytoplanctonique supplémentaire dans le modèle afin d'obtenir en été des pics d'amplitude élevée. Nous avons différencié les algues par leur taux de croissance, leur température optimale de croissance, leur constante de demi-saturation en substrat et par la quantité de phosphore qu'elles contiennent.

\subsection{Résultats de la simulation de référence.}

Après plusieurs simulations, le meilleur ajustement est obtenu avec le jeu de paramètres dont les valeurs sont données dans le tableau page suivante.

Les résultats montrent que l'évolution du phytoplancton est assez bien représentée (fig. 16). On remarque que le pic printanier est déterminé par le réchauffement de l'eau, alors que les pics d'été et d'automne sont déclenchés par l'approfondissement de la thermocline qui met à disposition du phosphore dans la couche de surface. On note, en été, que le pic du zooplancton est décalé par rapport aux données mesurées. En effet, le modèle simule le système proie-prédateur qui implique par hypothèse une croissance du zooplancton consécutive à celle des algues, ce qui n'a pas été observé sur le terrain en 1986. 
Valeurs des paramètres retenues lors de l'ajustement

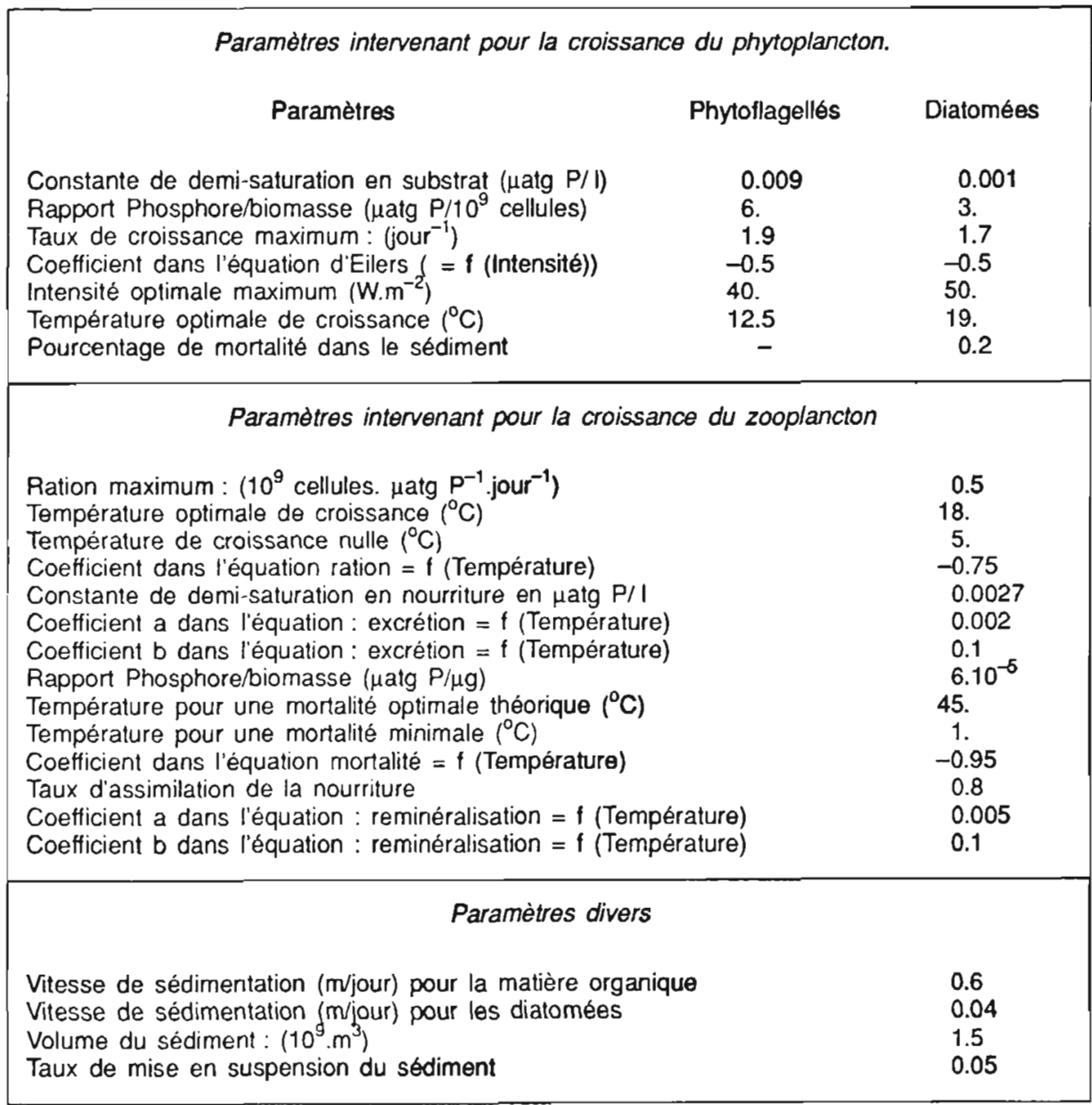

\subsection{Effet de quelques modifications de la structure du modèle.}

Afin de commencer à étudier l'effet d'une discrétisation plus fine de la verticale (par un couplage du modèle biologique à un modèle hydrodynamique vertical), quelques modifications de structures ont été apportées au modèle précédent dit "de référence": - introduction de la couche euphotique comme seule couche de production,

- migration du zooplancton limitée à $22 \mathrm{~m}$.

Ces deux modifications seront introduites d'abord séparément afin 


\section{SAINTE-CROIX ANNEES 1985 EI 1986}
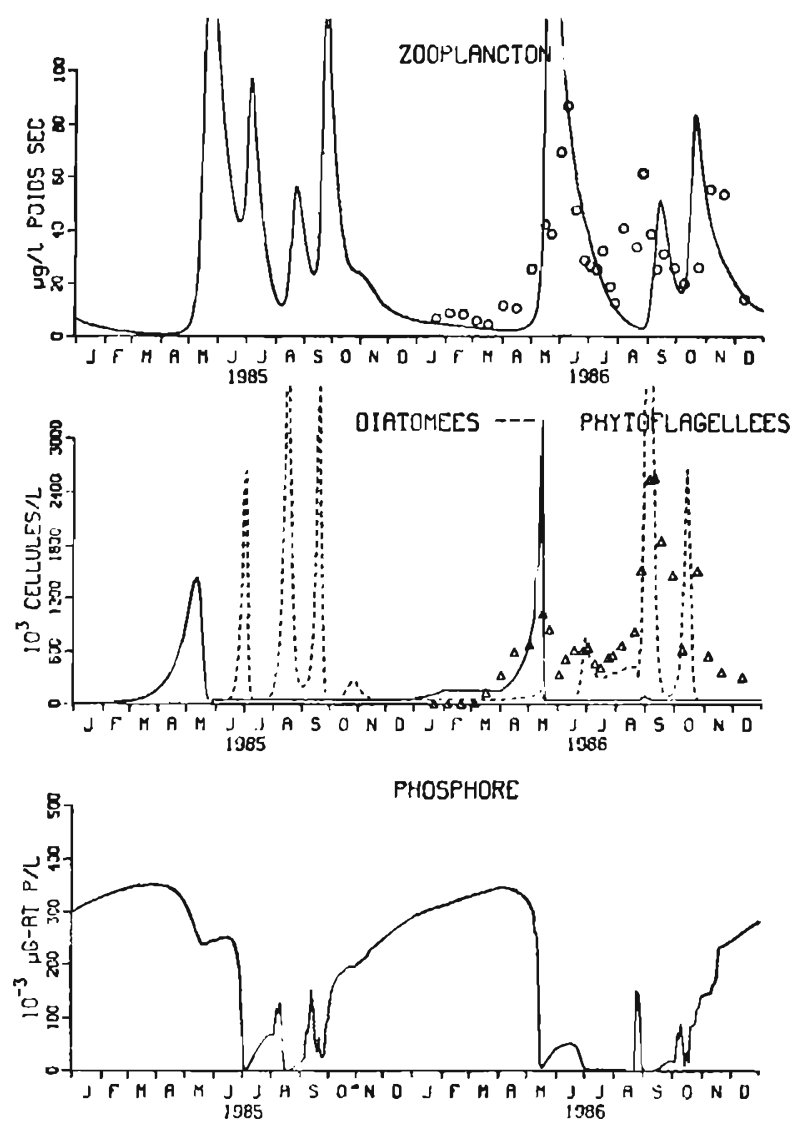

Fig. 16. - Simulation de l'évolution du plancton et du phosphore : modèle de référence.

de juger de leurs effets respectifs sur les résultats, puis simultanément, en gardant le jeu de paramètres de calage du modèle de référence.

Introduction de la couche euphotique.

Dans le modèle bicouche de référence, la production photosynthétique a lieu dans chaque couche avec un éclairement moyenné sur la hauteur de la couche. Cette structure est très pénalisante au printemps lorsque la stratification n'est pas bien établie : la couche de surface est alors très importante et l'éclairement moyen y est très faible.

En rajoutant une couche euphotique dont la hauteur est calculée à partir des mesures de profils de lumière, la production photosynthétique a lieu uniquement dans cette couche, avec un éclairement beaucoup plus réaliste. Cette troisième couche n'a été rajoutée que pour la production 


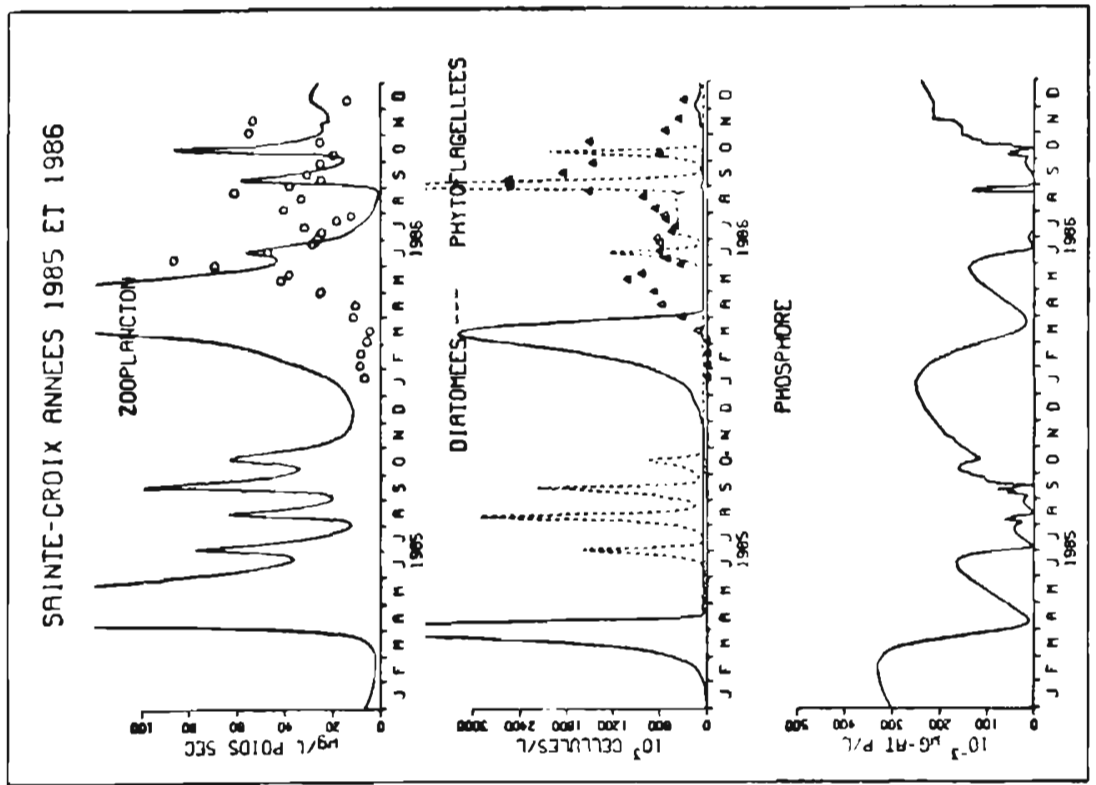

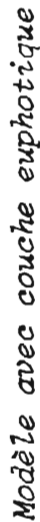
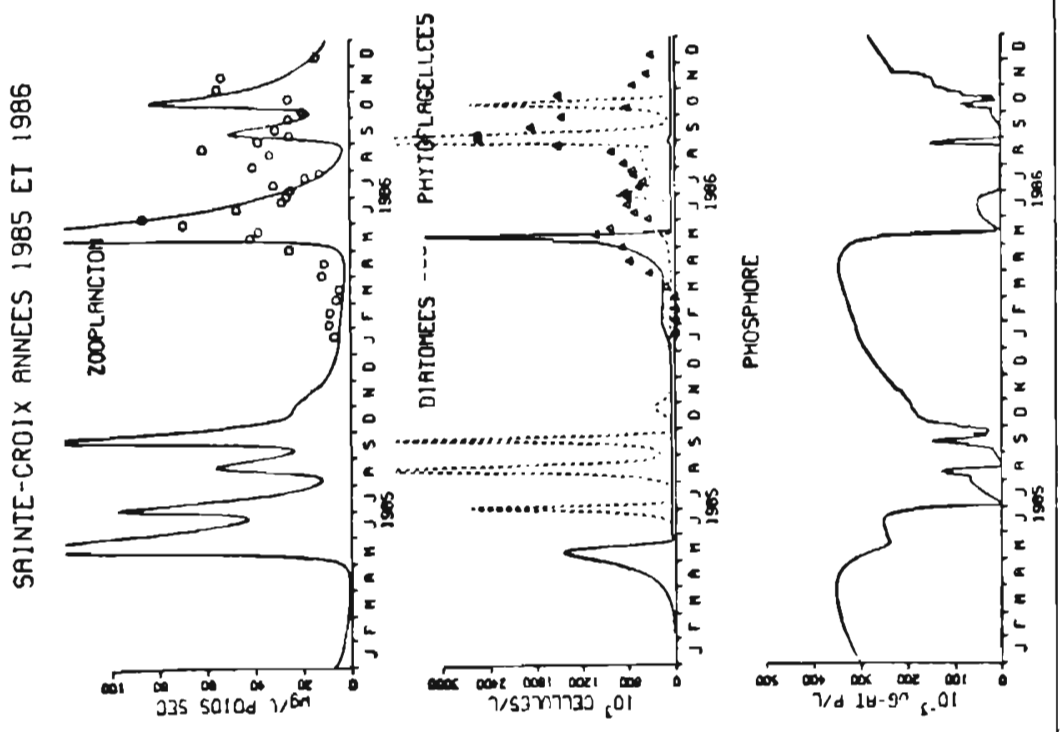

है 


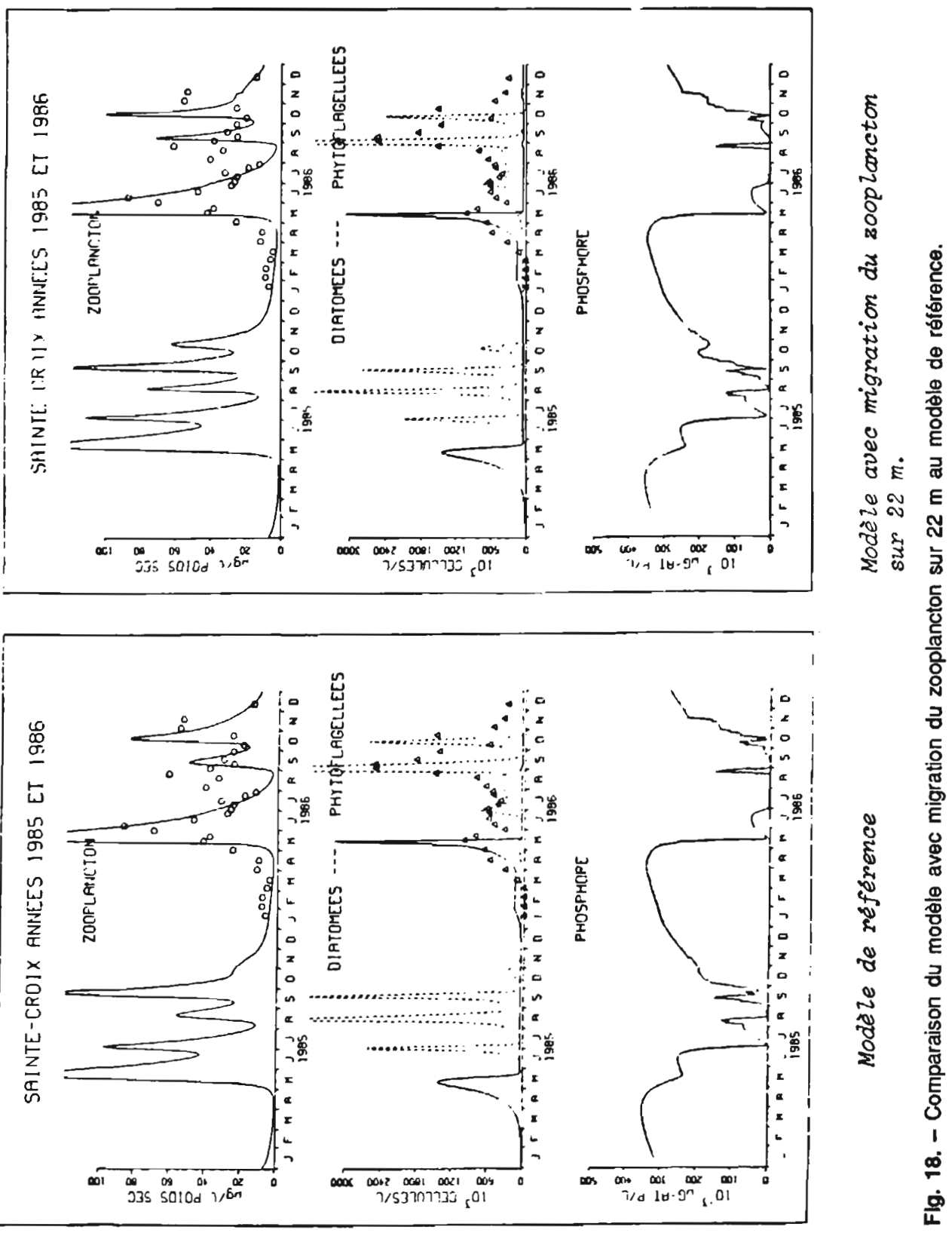


phytoplanctonique, la structure bicouche du modèle étant ensuite rétablie immédiatement par mélange.

Les résultats de la figure 17 montrent effectivement un démarrage phytoplanctonique très précoce en hiver si on introduit la couche euphotique, prouvant que la limitation de la croissance par la lumière est une fonction très sensible au forçage. L'influence en est moins sensible en été où la couche de surface est beaucoup plus proche de la couche euphotique.

\section{Migration du zooplancton limitée à $22 \mathrm{~m}$.}

Dans le modèle bicouche de référence, le zooplancton a une répartition verticale homogène sur les 2 couches, ce qui revient à considérer que la migration verticale a lieu sur toute la profondeur du lac, soit $80 \mathrm{~m}$. Les prélèvements zooplanctoniques réalisés in situ montrent que la migration verticale est limitée à $22 \mathrm{~m}$. Après l'introduction de cette modification, les résultats (fig. 18) sont assez peu modifiés. Le zooplancton est un peu plus dynamique, avec des pics de plus grande amplitude et plus courts. Les pics phytoplanctoniques sont légèrement réduits en amplitude mais on ne constate aucun décalage important dans le temps.

Introduction simultanée de la couche euphotique et de la migration du zooplancton sur $22 \mathrm{~m}$.

L'introduction de ces deux modifications simultanées a un effet très sensible au printemps. Le pic phy- toplanctonique démarre très tôt et n'est pas contrôlé par le zooplancton qui, bien que plus dynamique, n'a un broutage effectif que sur $22 \mathrm{~m}$. En été, les résultats (fig. 19) sont très peu modifiés : les 2 pics zooplanctoniques sont de plus faible amplitude et plus étalés induisant des pics phytoplanctoniques légèrement plus forts et étalés.

\section{Réflexions sur les résultats.}

Ces deux essais montrent à quel point la réponse de l'écosystème est sensible à la structure hydrodynamique choisie: la dimension verticale représente dans un lac l'essentiel des phénomènes et il est important de bien la représenter, à l'échelle de temps et d'espace des processus biologiques étudiés. De plus, il apparaît que le jeu des paramètres biologiques donnant le meilleur ajustement, "contient" implicitement l'information de la structure physique choisie. II est donc capital de ne jamais dissocier un jeu de paramètres de modélisation des hypothèses choisies pour réaliser le modèle.

\subsection{Analyse de sensibilité.}

Pour mieux connaître la réponse de l'écosystème et pour mettre en évidence l'influence des paramètres intervenant dans le modèle, il est nécessaire de réaliser une étude systématique dans laquelle le poids de chaque paramètre peut être quantifié. Nous ne pouvons pas nous permettre d'effectuer une telle étude sur l'ensemble des paramètres en raison du 


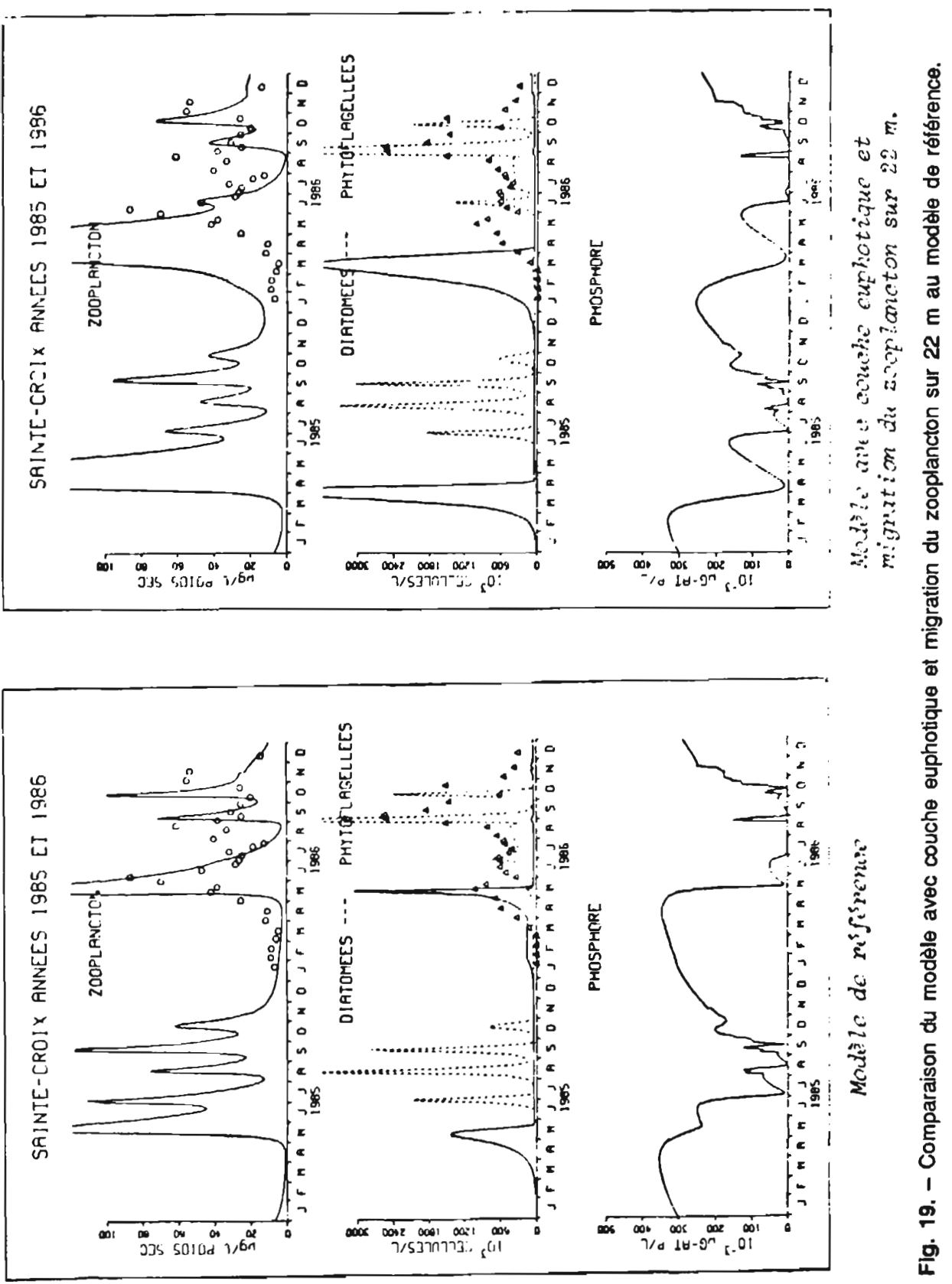


grand nombre d'essais que cela implique.

Nous avons sélectionné un nombre restreint de paramètres biologiques qui nous paraissent importants : température optimale de croissance, constante de demi-saturation en nutriments, taux de croissance, rapport phosphore/biomasse pour les algues et coefficient de mortalité pour le zooplancton. De plus, nous complétons l'étude de sensibilité en testant le pas d'intégration ainsi que le coefficient d'extinction de la lumière.

Nous avons choisi une méthode simple qui nous permet de visualiser l'impact d'un paramètre sur l'écosystème : nous comparons le système de référence à un système perturbé par les variations d'un paramètre. Nous utilisons la représentation graphique de Thornton (1979) (cité par Garçon, 1980), qui utilise le cercle comme représentation annuelle.

Nous représentons sur une année le rapport existant entre la concentration d'une variable perturbée (par les variations d'un paramètre) et la concentration de référence de cette même variable. Chaque point est représenté en coordonnées polaires: l'angle du vecteur correspond au jour de l'année et sa longueur à la valeur du rapport des concentrations.

Cette méthode, très sensible, permet de détecter des différences de concentration très faibles mais sans en préciser la nature : décalage du pic ou changement d'amplitude.

Pour visualiser l'évolution des concentrations des variables au cours de l'année, nous complétons cette première représentation graphique en traçant directement la concentration des variables avec 'le système de référence (courbe verte) et le système perturbé (courbe rouge). Cette représentation permet de distordre les échelles: les variations d'amplitude sont réduites au profit des variations temporelles. Si les faibles différences de concentration ne sont pas visibles, en revanche, un décalage de quelques jours est facilement détectable. Nous fixons, pour chaque variable, comme rayon minimum la concentration nulle et comme rayon maximum la concentration maximale observée.

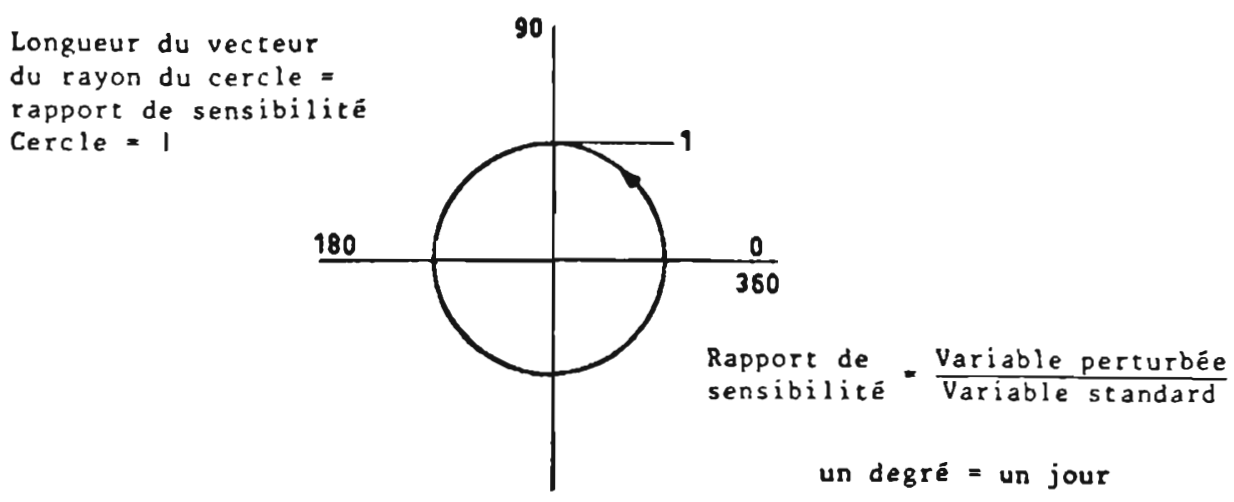


ANFLYSE DE SENSIBILITE A LA YAL FIR D COEFFICIENT D'EXTINCTION DE LA LUMIERE

EVOLUTSON DU RAPPORS DES CONCENTRATIONS YALEUR DE REFERENCE CK : YFRIALLE
$a-0.15$.
$c x-0.25$
cK- 0.35
CK- 0.5
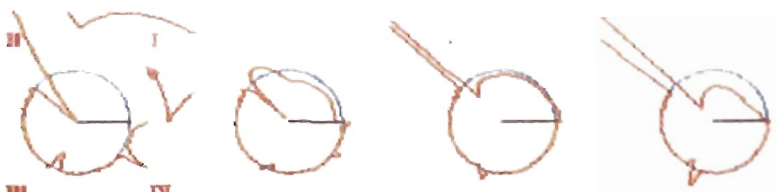

PHOTORLELULS
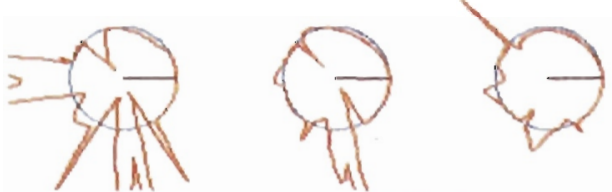

as proers
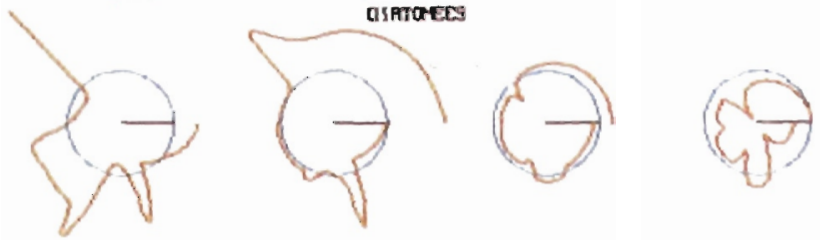

ZOTPLAMTHON
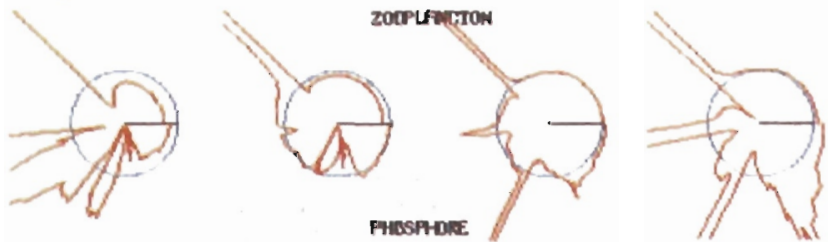

AWALSE DE SENSIBILITE A LA VALEUR OU COEFF ICIENT D'EXTINCTION DE LA WUHIERE CONPARASSON DES CRHONOLOQIES YALEUR DE REFERENCE CK : YTRIAELE

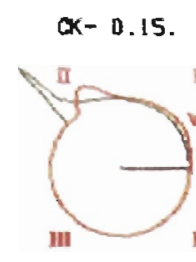
$c x-0.25$

CK- 0.35

CK- 0. IS
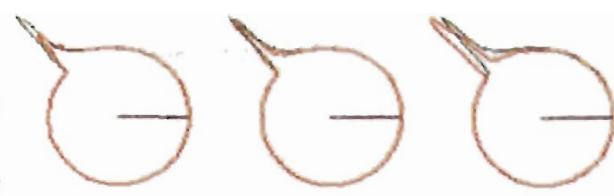

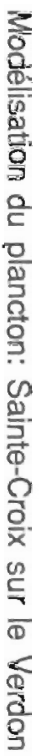
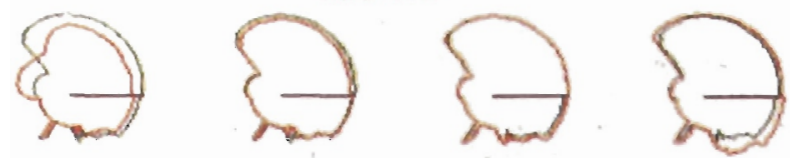

PHISPMDRE

mortorideus
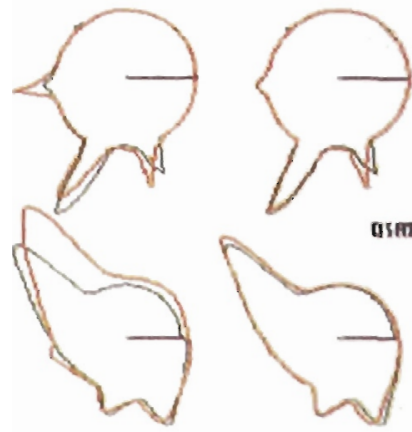

arnorea
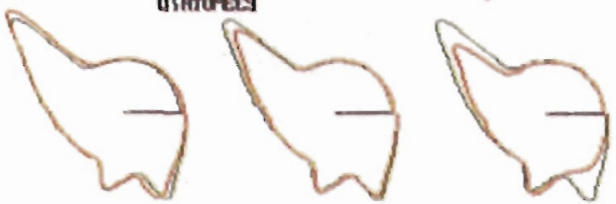

zotourwitow
Fig. 20. - 

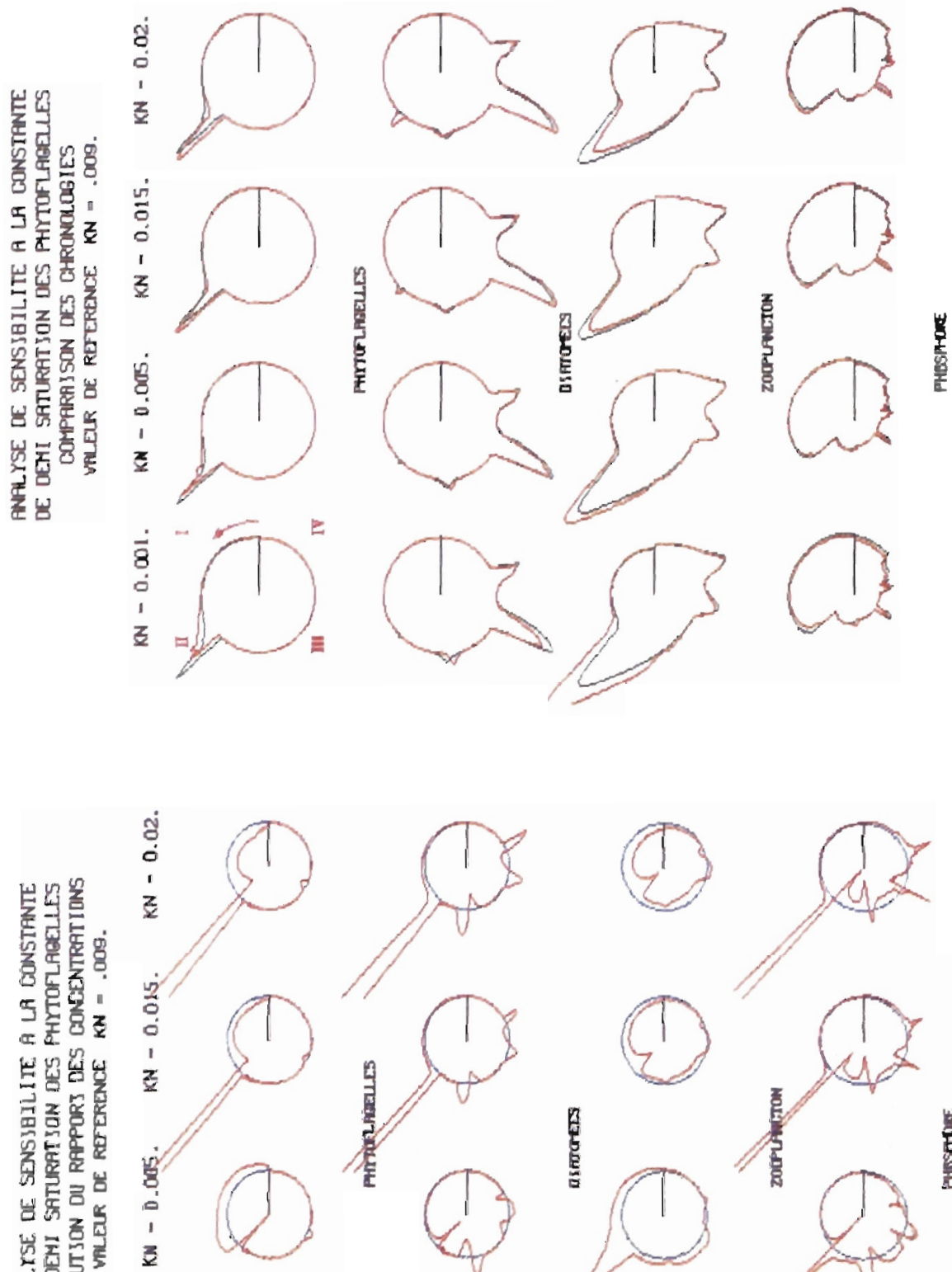
重嵒要
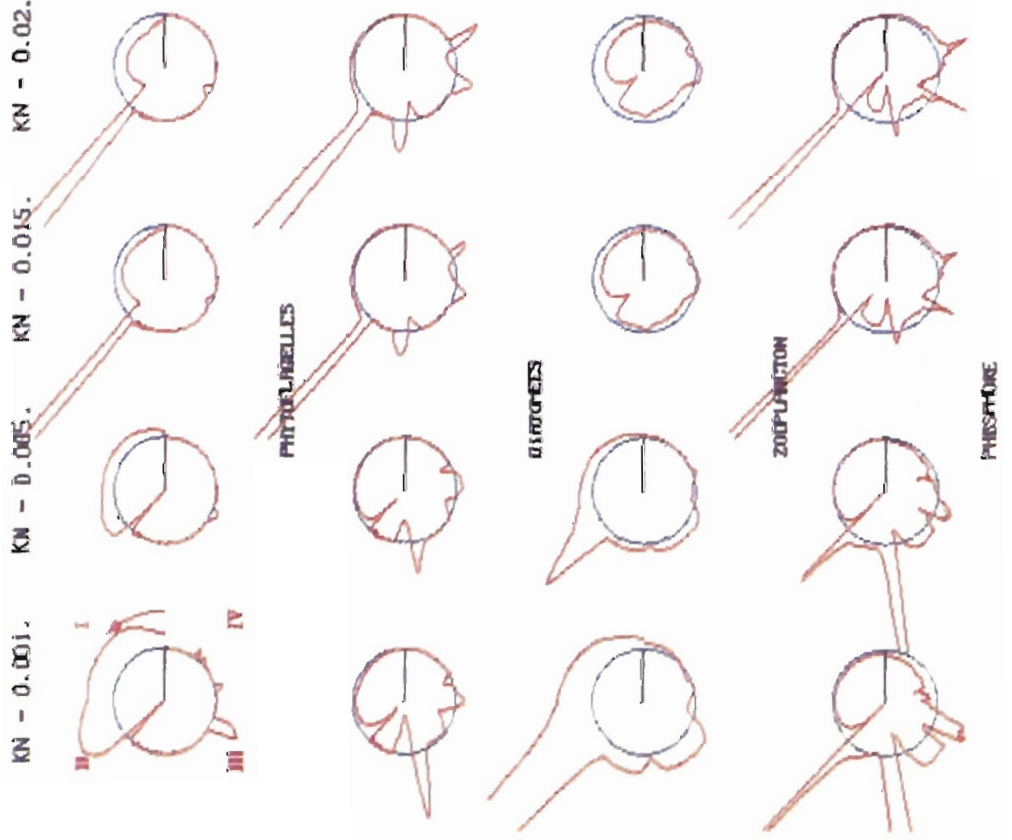

1
ㅁ
$\frac{0}{4}$ 


\section{Influence du pas de calcul.}

Nous avons testé 4 valeurs du pas de calcul allant de 7 minutes à 12 heures (fig. 21). L'évolution des variables n'est quasiment pas modifiée excepté lorsque le pas de temps est de 12 heures. En effet, ce pas d'intégration est trop grand lorsque les gradients deviennent importants. Par contre, il n'est pas nécessaire d'utiliser un pas de temps trop petit étant donné la similitude des réponses du système.

\section{Influence du coefficient d'extinction de la lumière.}

Une faible valeur du coefficient d'extinction entraîne une croissance plus forte du phytoplancton et du zooplancton (fig. 20). Les valeurs extrêmes $(0.15$ et 0.45$)$ perturbent surtout la concentration du zooplancton en raison des décalages des pics des algues. Plus le coefficient d'extinction est faible, plus les pics des phytoflagellés et des diatomées se produisent tôt. C'est la valeur $0.35 \mathrm{du}$ coefficient qui perturbe le moins le système. Cette valeur est une bonne moyenne des valeurs observées sur le site de Sainte-Croix.

Cette variable semble importante pour l'écosystème et les résultats suggèrent qu'en l'absence de mesures chronologiques, il faut connaître une bonne estimation de la moyenne annuelle.

Influence des paramètres biologiques.

Nous présentons ici les principales conclusions de l'étude de sensibilité des paramètres biologiques figurant en annexe 6 du rapport Pourcher et Salençon, 1988.

- Un changement assez faible des paramètres caractérisant les phytoflagellés perturbe la croissance des diatomées de façon non négligeable. Par contre, les phytoflagellés sont peu sensibles aux variations des paramètres des diatomées.

- Les variations des paramètres intervenant sur la croissance des algues provoquent un décalage des pics sans trop en modifier l'amplitude.

- Certains jeux de paramètres entraînent les mêmes effets sur les variables, ce qui s'explique par le fait qu'ils aient les mêmes conséquences sur les fonctions du système. Ainsi, une température optimale de $19.5^{\circ} \mathrm{C}$ augmente la croissance des phytoflagellés en hiver, au même titre qu'un taux de croissance de $2.3 \mathrm{j}^{-1}$ et qu'une constante de demi-saturation égale à $0.02 \mu \operatorname{atg} P / /^{6}$, les autres paramètres étant, par ailleurs, égaux aux valeurs de référence.

- Le zooplancton apparaît comme un régulateur de la croissance des algues: un changement de ses paramètres, mème faible, a des conséquences importantes sur l'écosystème (fig. 22). Nous voyons ici le rôle primordial joué par le broutage du zooplancton et l'on peut se poser la question de la fermeture du système au niveau du zooplancton: n'est-il pas nécessaire, dans certains cas, de rajouter une variable d'état supplémentaire simulant le niveau trophique supérieur? 

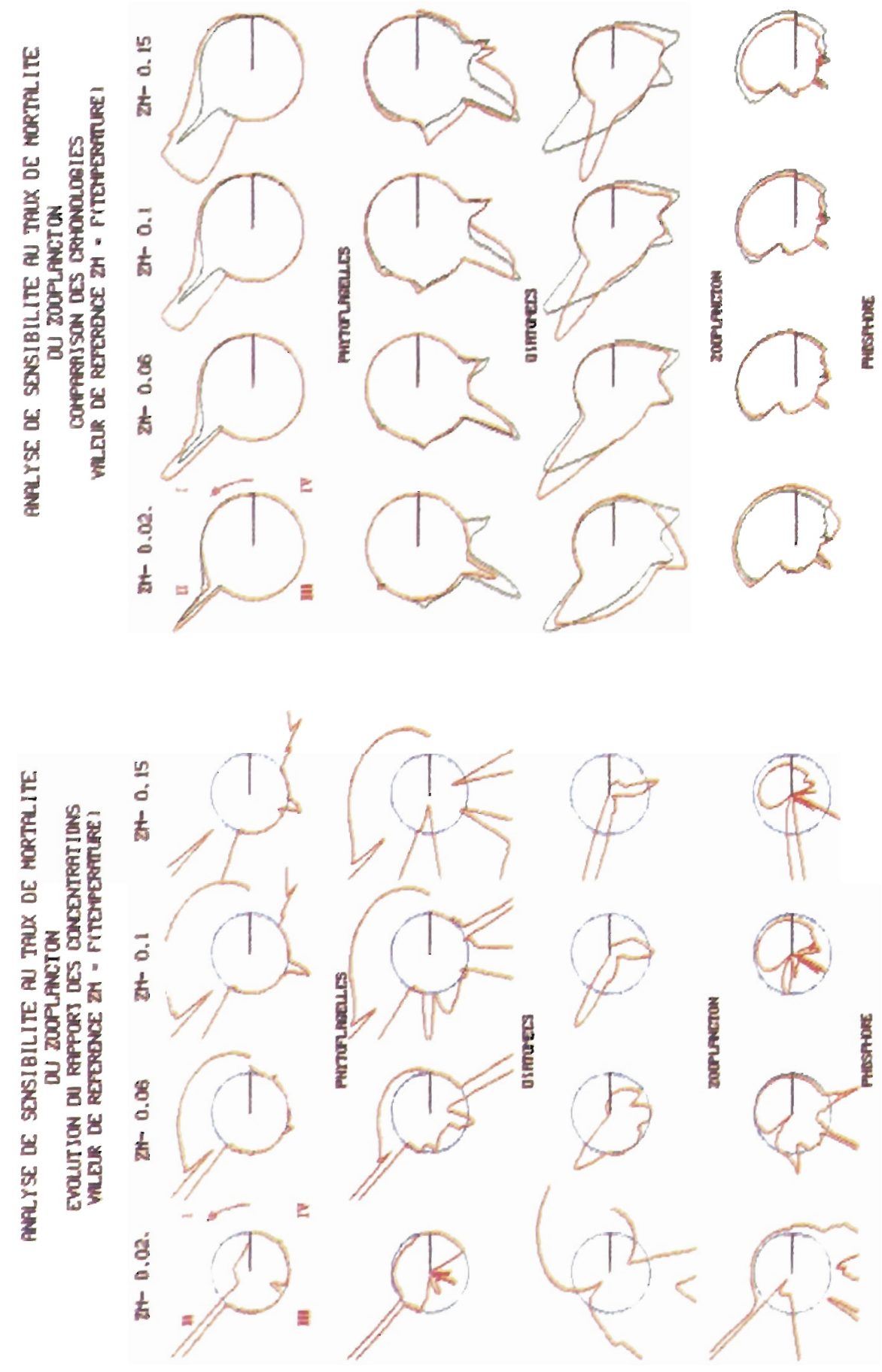

ลี่
훈 


\section{CONCLUSION}

Un modèle d'écosystème de résenvoir a été mis au point pour la retenue de Sainte-Croix.

Ce modèle biologique s'appuie sur une structure physique simple (modèle bicouche) qui représente la stratification thermique saisonnière à partir de variables de forçage.

Le modèle simule l'évolution de 5 variables d'état:

- la biomasse de deux catégories de phytoplancton,

- la biomasse d'un groupe de zooplancton,

- la concentration en un élément nutritif: le phosphore,

- la concentration en matières organiques.

La période de simulation s'étend du $1^{\text {er }}$ janvier 1985 au 31 décembre 1986. L'ensemble des données des deux années n'étant pas disponible, le modèle a été ajusté pour l'année 1986. La validation n'a pu être réalisée pour l'instant.

Ce modèle simple a permis de reproduire de façon satisfaisante les principaux événements annuels de l'écosystème: trois poussées phytoplanctoniques et zooplanctoniques successives, au printemps, en été et en automne.

Si un groupe zooplanctonique a suffi, en revanche, il a fallu considérer 2 espèces d'algues de caractéristiques différentes, notamment pour la température optimale de croissance et la préférence nutritionnelle. Cette différenciation a permis de reproduire une compétition interspécifique, cha- que poussée phytoplanctonique étant dominée par un seul groupe.

Afin d'estimer l'importance des paramètres de la modélisation, une étude de sensibilité a été réalisée sur 11 des paramètres biologiques les plus importants.

Le modèle est très sensible à de faibles variations des paramètres, mettant en évidence l'importance de leur choix.

Les paramètres du zooplancton entraînent les perturbations les plus importantes sur la réponse du modèle: c'est lui qui ferme le système en représentant le niveau trophique supérieur. Son rôle primordial dans le système suggère que, dans certains cas, il serait nécessaire de simuler la prédation à un niveau supérieur.

On note également l'importance des variables physiques sur le système proie-prédateur :

- l'influence de la température sur la dynamique des processus de croissance biologique est primordiale;

- le phénomène de stratification thermique, en séparant la masse d'eau en 2 couches quasiment indépendantes l'été, joue un rôle déterminant sur le stock des nutriments disponibles dans la couche euphotique.

L'importance de la structure thermique verticale implique de disposer d'une chronique de mesures de température sur la verticale à au moins deux niveaux situés dans l'épilimnion et dans l'hypolimnion. Elle justifie d'autre part, pour certaines études, 
une simulation numérique du régime thermique.

L'influence de la structure physique du modèle sur la réponse de l'écosystème a été étudiée. II apparaît que l'écosystème est très sensible à une représentation physique pius fine de la verticale, et que les paramètres biologiques donnant le meilleur ajustement sont très fortement liés à cette structure.

Si l'on souhaite obtenir un modèle cohérent, il importe donc d'équilibrer la complexité des sous-modèles physiques et biologiques.

C'est en effet leur couplage qui apportera une réelle amélioration dans la compréhension et la représentation de l'écosystème.

\section{BIBLIOGRAPHIE}

Agoumi A., 1985. Modélisation de l'écosystème pélagique en Manche: Etude de l'influence des phénomènes physiques sur le système planctonique. Thèse de doctorat d'état es Sciences Naturelles.

Bin-Mole R., Pont D., Vaquer A. 1986. Répartition spatiale du phytoplancton dans le réservoir de Sainte-Croix (Var) en période de stratification estivale Sciences de l'eau, 5 (1986), p. 101 . 105.

Chahuneau F., Desclers S., Meyer J.A. 1980. Les modèles de simulation en écologie lacustre. Présentation des différentes approches et analyse des modèles existants. Acta Oecologica, Oecologica generalis, 1980, vol. 1, $n^{\circ} 1$, p. 27-50.

Chahuneau F., Desclers S., Meyer J.A. 1981. Simulation du cycle du phos- phore dans le lac de Nantua. Oceanis, vol. 6, Fasc. 1 (1980-1981), p. 99-108.

Dauta, A. 1983. Conditions du développement du phytoplancton : Etude comparative du comportement de huit espèces en culture; cinétique d'assimilation et de croissance : étude expérimentale; modélisation appliquée aux cultures et à un milieu naturel: le Lot. Thèse de doctorat d'état es Sciences.

Dauta A., 1983. Conditions du développement du phytoplancton. Modélisation de cultures d'algues. Une approche des conditions de succession. Annales limnologiques, vol. 19 (2), 1983, p. 7386.

Elhasa M., 1987. Bilan des apports de sels nutritifs au lac de Sainte-Croix (Provence) et leur utilisation par le phytoplancton dans la retenue. Thèse de $3^{e}$ cycle (Université de Provence).

Enderle M.J., 1982. Impact of a pumped storage station of temperature and water quality of the two reservoirs. ISEM Journal, vol. 4, 1982, $n^{\circ}$ 3-4.

Enderle M.J., Gras R.A., 1983. Les différents mécanismes hydrauliques gérant la croissance du plancton dans une retenue: Action du vent, des apports d'eau, des soutirages. Texte de communication présenté au $X X^{e}$ congrès de l'AIRH - Moscou.

Garçon V., 1981. Modélisation numérique d'un écosystème aquatique. Application au résenoir de Grangent sur la Loire. Bulletin de la Direction des Etudes et Recherches, série $A, n^{0} 3$, 1981.

Grégoire A. 1982. Contribution à l'étude hydrobiologique d'une rivière aménagée : le Verdon. Les lacs de barrage et les tronçons de cours d'eau à débit régulé. Cahiers du laboratoire d'hydrobiologie de Montereau, $n^{\circ} 13$.

Jorgensen S.E., 1979. Handbook of Environmental Data and Ecological Parameters International Society for Ecological Modelling. 
Kremer J.N., Nixon S.W. 1978. A Coastal Marine Ecosystem. Simulation and Analysis. Ecological Studies 24, Springer-Verlag Berlin Heidelberg New York, 1978.

Lehman J.T., Botkin D.B., Likens G.E., 1975. The assumptions and rationales of a computer model of phytoplancton population dynamics. Limnology and oceanography, vol. 20 (3), 1975.

Patten B.C. 1975. Systems Analysis and Simulation in Ecology, vol. III, Academic Press, Inc. New-York.

Pourcher A.M., Salençon M.J., 1988. Modélisation d'un lac oligotrophe : SainteCroix. Rapport EDF - DER - HE/31 88.47.

Salençon M.J. 1986. Modélisation de l'eutrophisation des lacs et retenues. Communication présentée au Symposium sur la qualité des eaux de surface, Casablanca (Maroc), novembre 1986.
Thébault J.M., 1984. Modélisation des premiers niveaux du réseau trophique pélagique marin. Mise au point de modules et simulation de séries expérimentales. Thèse de doctorat de $3^{\mathrm{e}}$ cycle.

Thébault J.M., 1985. Etude expérimentale de la nutrition d'un copépode commun (Temora Stylifera Dana). Effets de la température et de la concentration de nourriture. J. Exp. Mar. Biol. Ecol. 1985 , vol. 93, p. 223-234.

Wang-Ming P., Harleman D.R.F., 1982. Hydrothermal - Biological coupling of lake Eutrophication models. Report number 270 of the Massachussetts Institute of Technology.

Waterloopkundig Laboratorium, 1979. Light, phatosynthesis and carbon/chlorophyll ratios. Amsterdam University limnological laboratory. Report 1310 (2), 1979. 